This document is the accepted manuscript version of the following article:

Bü1bü1, E., Rupper, P., Geue, T., Bernard, L., Heuberger, M. P., \& Hegemann, D. (2019). Extending the range of controlling protein adsorption via subsurface architecture. ACS Applied Materials and Interfaces. https://doi.org/10.1021/acsami.9b14584

\title{
Extending the range of controlling protein adsorption via subsurface architecture
}

Ezgi Bülbüll, 2, Patrick Rupperl, Thomas Geue ${ }^{3}$, Laetitia Bernard ${ }^{4}$, Manfred Heuberger ${ }^{1,2}$ and Dirk Hegemann ${ }^{* 1}$

${ }^{1}$ Laboratory for Advanced Fibers, Empa, Swiss Federal Laboratories for Materials Science and Technology, 9014 St. Gallen, Switzerland

${ }^{2}$ Laboratory for Surface Science and Technology, Department of Materials, ETH Zurich, 8093 Zurich, Switzerland

${ }^{3}$ Laboratory for Neutron Scattering and Imaging, Paul Scherrer Institute, 5232 Villigen PSI, Switzerland

${ }^{4}$ Laboratory for Nanoscale Materials Science, Empa, Swiss Federal Laboratories for Materials Science and Technology, 8600 Dübendorf, Switzerland

KEYWORDS: subsurface modification, plasma polymerization, BSA adsorption, hydration, gradient film

\section{Corresponding Author}

*dirk.hegemann@empa.ch 


\begin{abstract}
Recently, it has been shown that water, confined in a plasma polymer subsurface chemical gradient, nanometers below the surface, significantly reduced the amount of adsorbed protein bovine serum albumin (BSA). Relating to this effect, we proposed the hypothesis that oriented water molecules within the subsurface gradient generate a long-range dipolar field, which interacts with dipolar proteins such as BSA near the surface region. This study extends the above used insitu multistep plasma deposition process to introduce plasma oxidation modifications of the subsurface architecture with the aim to further control the effect on protein adsorption.

Neutron reflectivity measurements reveal that the oxidation time increases the amount of matrixconfined water. There is, however, an optimal oxidation time to obtain minimal protein adsorption, which suggests that a minimal distance between confined water molecules plays an important role. Altogether we can extend the range of controlling the adsorbed protein mass by the introduction of this additional plasma oxidation step.
\end{abstract}




\section{Introduction}

Introducing chemical gradients is a promising new strategy to tailor surface properties, particularly in contact with fluid environments ${ }^{1,2}$. Materials comprising surface chemical gradients already found their use in diverse fields of materials science including biosensors, biomaterials, tissue engineering, and microelectronics ${ }^{3,4}$. It is interesting to note that nature uses similar concepts, for example a cell membrane, which has a hydrophilic surface and a hydrophobic subsurface.

We are particularly interested in vertical gradients that are defined by a gradual change of one or more properties with film depth, e.g. density, porosity, chemistry or wettability. Recent reports have described unexpected long-range effects that change surface properties while originating nanometers below the surface ${ }^{5,6}$. A physically and chemically stable vertical gradient architecture can be designed, comprising a hydrophilic to hydrophobic transition in the subsurface. Plasma enhanced chemical vapor deposition (PECVD) is a technique that is well suited to form such vertical gradient structures by variation of the deposition conditions in an in-situ multistep process $5,7,8$. Plasma polymerization is based on the plasma activation of organic compounds, the monomer, in the gas phase yielding a "plasma polymer" deposit. One can vary the properties of such films during growth over nanometers; e.g. chemical composition, thickness, hydrophilicity, density or cross-linking. Parameters that influence plasma deposition conditions include the type of plasma source, applied power and pressure, as well as input gas flow rates. Compared to conventional polymer films, plasma polymerized films embrace amorphous, randomly terminated and highly branched network structures ${ }^{9-11}$. While the local structure is randomized, the overall stoichiometric ratios are fixed. The resulting insoluble, mechanically tough and thermally resistant films enable applications in a variety of different fields, e.g. as a protective ${ }^{12}$, biocompatible ${ }^{13}$, anti-reflective ${ }^{14}$, and non-fouling films ${ }^{15}$. Thanks to the physical power input, chemical reactions can be forced that would not occur under wet chemical conditions; thus a wider range of monomers can be used. Notably, organosilicon-based monomers, in particular, HMDSO (hexamethyldisiloxane), $\left(\mathrm{CH}_{3}\right)_{3}$-Si-O-Si- $\left(\mathrm{CH}_{3}\right)_{3}$, are popular ${ }^{16}$. Depending on the applied power, the fragmentation of the monomer is driven by the physical breakage of $\mathrm{C}-\mathrm{H}$ and $\mathrm{Si}-\mathrm{CH}_{\mathrm{x}}$ bonds (3-5 eV), but also of the stronger Si-O-Si (6-9 eV) bond, the latter yielding highly film-forming species ${ }^{10}$. The further cleavage of methyl groups $\left(-\mathrm{CH}_{3}\right)$ at the surface or in the gas phase helps to constitute stable Si-O-Si bonds and $-\mathrm{CH}_{2}$ - linkages by densification/cross-linking ${ }^{10}$. With the controlled retention of methyl groups, hydrophobic and well cross-linked polydimethylsiloxane (PDMS)-like SiO:CH films are obtained.

A more hydrophilic type of layer can be obtained upon oxygen dilution of the HMDSO-feed into the plasma. Oxygen facilitates the dissociation of the HMDSO molecule; and oxidation of 
hydrocarbons is able to produce more hydrophilic, denser and hence more inorganic quartz-like $\left(\mathrm{SiO}_{2}\right)$ film characteristics.

Numerous research papers have discussed the functionality, the stability and the application of different variants of homogenous siloxane-based plasma polymer films (PPFs) 9, 10, 17, 18. The variation of the film properties from organic to inorganic has namely been studied for adhesion promotion and enhanced barrier properties using vertical gradients 18, 19. More recently, we introduced an in-situ two-step process including the formation of a hydrophilic, nanoporous base layer that is subsequently covered by a very thin hydrophobic terminal layer forming a vertical chemical gradient at their interface. As a notable finding, the protein adsorption using bovine serum albumin (BSA) was modified on the hydrophobic surface in the presence of such subsurface hydrophilic-to-hydrophobic gradients ${ }^{5,6}$. It was hypothesized that reduced protein adsorption is caused by water molecules confined in this subsurface vertical gradient. As already known in the literature, water molecules interacting with solid matrices mainly via hydrogen bonding could be in different states allowing dipolar reorientation, which depends sensitively on the surrounding chemical environment and thus energetic conditions. As a recently discussed example, water can form a ferroelectric phase when confined at increased distance between molecules as seen in a confining matrix ${ }^{20}$. If the confined water is ferroelectric, it is expected to interact with external electric fields with a chemical gradient in the matrix, or with a nearby dipolar protein.

Likewise, the investigated subsurface architecture can be considered as a matrix supporting the confinement and orientation of intruding water molecules during hydration. To further investigate the role of the hydrophilic-to-hydrophobic gradient at the interface, an additional plasma oxidation step was introduced to strengthen the gradient and ultimately extend the range of controlling protein adsorption.

\section{Methods}

\section{Plasma Polymer Deposition with a Vertical Gradient Structure}

Plasma polymer films (PPFs) were deposited using a capacitively coupled, asymmetric reactor. A radio frequency $(\mathrm{RF})$ driven electrode $(f=13.56 \mathrm{MHz})$ with an area of $21 \times 70 \mathrm{~cm}^{2}$ was mounted inside the reactor vessel with $8 \mathrm{~cm}$ distance from the (larger) chamber wall. The monomer hexamethyldisiloxane (HMDSO) (purchased from Fluka), the carrier gas Ar and the reactive gas oxygen (purchased from Carbagas, Switzerland) were introduced into the reactor by a gas showerhead facing the entire electrode area and supplying a uniform gas inlet. The liquid 
compound HMDSO was vaporized at reduced pressure and a temperature of $40^{\circ} \mathrm{C}$ and fed to the reactor via a thermostabilized mass flow controller $\left(43^{\circ} \mathrm{C}\right)$. In this work, five of the different PPFs were produced according to a previously described protocol 5, i) a $50 \mathrm{~nm}$ hydrophobic, hydrocarbon-rich film ( $\mathrm{SiO}: \mathrm{CH}$ ) deposited from the gaseous mixture of $4 \mathrm{sccm}$ (standard cubic centimeter per minute) vaporized HMDSO and $20 \mathrm{sccm}$ Ar using a power input of $50 \mathrm{~W}$, referred to as "SiO:CH" film, ii) a $50 \mathrm{~nm}$ hydrophilic, hydrocarbon-poor film $\left(\mathrm{SiO}_{\mathrm{x}}\right)$ deposited from the additional admixture of $40 \mathrm{sccm}$ oxygen to the HMDSO/Ar plasma using a power input of $100 \mathrm{~W}$, called " $\mathrm{SiO}_{\mathrm{X}}$ ", iii) a vertical gradient film consisting of a $50 \mathrm{~nm}$ thick $\mathrm{SiO}_{\mathrm{x}}$ base layer followed by the deposition of a nominally $4 \mathrm{~nm}$ thick SiO:CH terminating layer, called "Vgrad", and introducing the intermediate plasma oxidation step $-i v-v$ ) enhanced vertical gradient films by oxidizing the SiOx base layer for $1 \mathrm{~min}$ and $5 \mathrm{~min}$, respectively, prior to the deposition of the hydrophobic cover layer, referred to as "Vgrad-ox1" and "Vgrad-ox5". Such five PPFs were subject to full characterization including BSA adsorption, while two additional gradient films were prepared in the same way with 0.5 and 3 min intermediate plasma oxidation to extend the data set for protein adsorption measurements. For the gradient coatings, the plasma was shut off in between the deposition of the two siloxane layers and the post-oxidation step in order to adjust power and gas composition. Pressure was maintained in-situ at $7 \mathrm{~Pa}$ for all coatings. The substrates were cleaned by $\mathrm{Ar} / \mathrm{O}_{2}$ plasma ( $80 \mathrm{sccm} / 20 \mathrm{sccm}$ ) at $200 \mathrm{~W}$ applied power and $10 \mathrm{~Pa}$ pressure prior to the deposition step sequence. The deposited film density was determined from the measured volume and the mass of the film using 8 times thicker coatings on glass slides. Moreover, the film thickness was measured along a masked film edge on silicon wafers by a profilometer (Veeco, Dektak 150).

For quality assurance, the emission from the $\mathrm{HMDSO} / \mathrm{Ar}$ and $\mathrm{HMDSO} / \mathrm{O}_{2} / \mathrm{Ar}$ plasmas as well as from the $\mathrm{Ar} / \mathrm{O}_{2}$ pretreatment was characterized over the wavelength range of 280-800 nm using Optical Emission Spectroscopy (OES, AvaSpecULS2048-USB2, Avnates, NL) as an in-situ plasma diagnostic tool.

\section{Water Contact Angle (WCA) and Atomic Force Microscopy (AFM)}

Static water contact angles of all samples were measured with water $\left(\mathrm{CHROMASOLV}^{\circledR}\right.$, for HPLC, Sigma-Aldrich) at 5 different positions on the film surfaces in ambient atmospheric conditions by means of a drop shape analyzer (DSA 25, Krüss).

The surface morphologies of the PPFs were imaged using atomic force microscopy FlexAFM V5 (from Nanosurf AG, Liestal, Switzerland), operated in dynamic mode with a pyramidal silicon tip purchased from Budgetsensors (Tap190Al-G, radius $<10 \mathrm{~nm}$, the resonance frequency of $190 \mathrm{kHz}$ 
and force constant of $48 \mathrm{~N} / \mathrm{m}$ ). The device was equipped with a C3000 controller. Topographic images were acquired from at least three different locations on each film with a scanning area of $5 \times 5 \mu \mathrm{m}^{2}$ and a pixel resolution of $512 \times 512$. All taken images were analyzed by means of the open-source software Gwyddion $2.45^{21}$.

\section{X-ray Photoelectron Spectroscopy (XPS)}

The film compositions were assessed by a Scanning XPS Microprobe (PHI VersaProbe II spectrometer, Physical Electronics). The system was equipped with a monochromatized Al K $\alpha$ radiation $(1486.6 \mathrm{eV})$ and a hemispherical electron analyzer. The analysis chamber pressure was approximately $5 \times 10^{-7} \mathrm{~Pa}$. Randomly chosen measurement positions were analyzed via a micro-focused X-ray beam with a diameter of $100 \mu \mathrm{m}(25 \mathrm{~W}$ at $15 \mathrm{kV})$ and the analyzer was operated in the fixed analyzer transmission (FAT) mode. Survey scan spectra, high resolution spectra and angle-resolved X-ray photoelectron spectra (ARXPS) were recorded for carbon C1s, oxygen $\mathrm{O} 1 \mathrm{~s}$ and silicon $\mathrm{Si} 2 \mathrm{p}$ for the different examined samples. Survey and high resolution spectra were conducted at a takeoff angle of $45^{\circ}$ with respect to the surface plane using pass energies of $187.85 \mathrm{eV}$ and $29.35 \mathrm{eV}$, acquisition times of $160 \mathrm{~ms}$ and $1.44 \mathrm{~s}$ per data point and step widths of $0.8 \mathrm{eV}$ and $0.125 \mathrm{eV}$, respectively. This photoemission angle was promoted to angles of $15^{\circ}, 30^{\circ}, 45^{\circ}, 60^{\circ}$, and $75^{\circ}$ for ARXPS, with a slight increase in the pass energy (458.70 eV) and the step width $(0.25 \mathrm{eV})$ inducing the acquisition time of $320 \mathrm{~ms}$ per data point. Because reproducible ARXPS spectra were obtained at the same sample position, the sample was hardly damaged. During the measurement, possible sample charging was neutralized using dual beam flux with a combination of $\sim 1 \mathrm{eV}$ energy electrons and $\sim 10 \mathrm{eV}$ energy positive Ar ions. Referencing the aliphatic carbon peak to $284.4 \mathrm{eV}^{22}$, the obtained spectra were rescaled. Casa XPS Version 2.3.16 (Casa Software Ltd., Teignmouth, UK) was employed for data treatment. For the estimation of atomic concentrations, the instrument specific sensitivity factors have been used. Deconvolution of XP spectra was achieved using the Gaussian-Lorentzian product function of 70\% Gaussian and 30\% Lorentzian and a Shirley-type background was subtracted from the XPS peak areas to calculate the atomic concentration. For the particular spectral fitting of Si envelope a method described elsewhere ${ }^{23}$ was applied with the following binding energies corresponding to different oxidation states of $\mathrm{Si}$ : $\mathrm{Si}(-\mathrm{O})(101.5 \mathrm{eV}), \mathrm{Si}(-\mathrm{O})_{2}(102.1 \mathrm{eV}), \mathrm{Si}(-\mathrm{O})_{3}(102.8 \mathrm{eV}), \mathrm{Si}(-$ $\mathrm{O})_{4}(103.4 \mathrm{eV})$. Here, the peak energies were restrained to within $\pm 0.5 \mathrm{eV}$ of the above mentioned values and the full width at half maximum of these four peaks was equalized.

XPS information depth (ID) varies with the take-off angle relative to the surface plane and inelastic mean free path (IMFP) of the elements according to the following formula ID $=3 \lambda \sin \theta^{24}$. 


\section{Time of Flight Secondary Ion Mass Spectrometry (ToF-SIMS)}

The chemical composition in the vertical direction of the sample surface was further investigated via ToF-SIMS performed on a ToF-SIMS.5 (ION-TOF, Germany) instrument. At first, the outermost surface of reference PPFs, namely SiOx and SiO:CH, were analyzed using a $25 \mathrm{keV}$ $\mathrm{Bi}_{3}{ }^{+}$primary ion beam on areas of $200 \times 200 \mu \mathrm{m}^{2}$, combined with an electron flow to compensate for surface charging. The high mass resolution spectra permitted to identify the molecular fragments being characteristic for each PPF reference. The careful scrutinizing of the fragments obtained and the assignment of the distinctive peak intensities led to the selection of the following representative fragments: $\mathrm{SiOH}^{-}(m / z=44.98), \mathrm{SiC}_{2} \mathrm{H}_{5} \mathrm{O}^{-}(m / z=73.03), \mathrm{SiH}_{2} \mathrm{O}_{3}^{-}(m / z=77.98)$. While the fragments $\mathrm{SiC}_{2} \mathrm{H}_{5} \mathrm{O}^{-}$and $\mathrm{SiH}_{2} \mathrm{O}_{3}{ }^{-}$are characteristic for PDMS-like and inorganic quartzlike $\left(\mathrm{SiO}_{2}\right)$ structures, $\mathrm{SiOH}^{-}$indicates the presence of porosity in the film structure. Thus, they were then used to detect the transition from the plasma polymerized $\mathrm{SiOx}$ base layer to the $\mathrm{SiO}: \mathrm{CH}$ cover layer in the gradient samples. In-depth chemical information, in other words, the evolution of molecular fragments through the vertical gradient, was gathered via layer by layer ablation of the gradient PPF using a dual beam mode of $\mathrm{Bi}_{3}{ }^{+}$(analysis beam, $100 \times 100 \mu \mathrm{m}^{2}$ ) and $500 \mathrm{eV}$ $\mathrm{Cs}^{+}$(sputtering beam, $300 \times 300 \mu \mathrm{m}^{2}$ ). Note that the sputter area was set so to provide a slow etching rate and ensure a homogenous surface analysis. Negatively charged secondary ions in the range from 1 to 320 mass units were detected with a sensitivity in the ppm range. The consistency of the depth profiles of each sample was ensured by performing analysis three times at randomly selected areas.

The full ToF-SIMS depth profiles for all abovementioned PPFs were acquired via sputtering the films until reaching the Si wafer substrate. Thus, an average sputtering rate for reference film layers of SiOx and $\mathrm{SiO}: \mathrm{CH}$ calculated as 0.055 and $0.13 \mathrm{~nm} \mathrm{~s}^{-1}$, respectively, based on the average sputtering time needed to reach the substrate for the corresponding film thickness $(\sim 50 \mathrm{~nm})$ as determined independently by profilometry. In this article, only the profiles acquired during the time between the seconds $0-60$ are discussed for the sake of resolving the transition zone of gradient films.

\section{Neutron Reflectometry (NR)}

For the NR measurement, vertical gradient PPFs with 1 and 5 min plasma oxidation (Vgrad-ox1 and Vgrad-ox5) were deposited on a double-side-polished silicon block ( $<100>, 1 \mathrm{~cm} \times 5 \mathrm{~cm} \times 10 \mathrm{~cm}$, SPM, Liechtenstein) implementing the above-stated protocol 
for plasma polymerization. For this purpose, a special sample holder, a metallic frame precisely fitting to the edges of the sample block, was designed to ensure the same homogeneous plasma deposition and oxidation conditions. NR measurements were carried out using the AMOR reflectometer at the Swiss Spallation Neutron Source (SINQ), at the Paul Scherrer Institute (PSI), Villigen PSI, Switzerland ${ }^{25,26}$. The reflectometer was designed to run in a time of flight mode in a range between 0.35 and $1.2 \mathrm{~nm}$ wavelength $(\lambda)$, allowing the recording of reflectivity $(\mathrm{R})$ data at the following angles of incidence $(\theta): 0.5^{\circ}, 1.5^{\circ}$, and $3.2^{\circ}$. Thanks to the energy dispersive instrumental feature of AMOR, the recorded reflectivity could be obtained in terms of the magnitude of momentum transfer vector $\vec{q}$, covering the range of $0.01 \AA^{-1}-0.20 \AA^{-1}$, which can

be calculated by $\mathrm{q}_{\mathrm{z}}=\left(\frac{4 \pi}{\lambda}\right) \sin \theta$. By dividing the intensity of the reflected beam to the intensity of the incoming beam and normalizing to 1 , the reflected intensity perpendicular to the reflecting interface was estimated. A distinct increase in scattering length density (q) by the usage of deuterated water (Sigma Aldrich, 99.9 atom \% D, $\mathrm{q}_{\mathrm{D}_{2} \mathrm{O}}=6.366 \times 10^{-6} \AA^{-2}$ ) rather than of normal water $\left(\mathrm{q}_{\mathrm{H}_{2} \mathrm{O}}=-5.610 \times 10^{-7} \AA^{-2}\right)$ allowed a better contrast between the PPF and the diffusing solution for the hydrated state measurements. After the measurement of all samples in dry state over the applied measurement time of 6 hours, the film surfaces were immersed in $\mathrm{D}_{2} \mathrm{O}$ for 6 hours and then the measurement was performed in solution for another 6 hours. Therefore, the time for water penetration was extended up to 12 hours.

The analysis of the measured data was performed on the basis of the Parratt formalism ${ }^{27}$ with Parratt32 software package ${ }^{28}$, which ensured the fitting of the reflectivity data as $\log \mathrm{R} v \mathrm{q}_{\mathrm{z}}$ using a gradient model. The PPFs were divided into several layers consisting of $\mathrm{Si}$ block, $\mathrm{SiO}_{2}$ native layer, $\mathrm{SiOx}$ and $\mathrm{SiO}: \mathrm{CH}$ plasma polymers, and lastly, the surrounding medium (air or $\mathrm{D}_{2} \mathrm{O}$ ) to estimate the scattering length density gradient. In the model, the scattering length density of $2.073 \times 10^{-6} \AA^{-2}, \quad 3.4 \times 10^{-6} \AA^{-2}, \quad 6.366 \times 10^{-6} \AA^{-2}$ were used for $\mathrm{Si}, \mathrm{SiO}_{2}$ and $\mathrm{D}_{2} \mathrm{O}$, respectively 25 .

\section{Protein Adsorption}

The adsorption of the model protein, bovine serum albumin (BSA, purchased from Sigma-Aldrich as a lyophilized powder, purity $\geq 96 \%$ ) on PPFs was observed using an optical technique, namely Transmission Interferometric Adsorption Sensor (TInAS), which is based on Fabry-Pèrot white light interference ${ }^{29,30}$. This high-speed sensor enables to carry out time-resolved measurements of molecular adsorption onto surfaces as well as offering additional information about adsorbate and adsorbent properties. Mimicking Fabry-Pèrot gradient configuration, a transparent sensor is 
composed of a clean glass substrate coated by a roughly $25 \mathrm{~nm}$ thin aluminum mirror layer and a $2.5 \mu \mathrm{m}$ thick $\mathrm{SiO}_{2}$ spacer layer exhibiting a distinctive refractive index in each layer. Upon molecular adsorption from the adsorbate solution, here BSA adsorption from phosphate-buffer saline (PBS) solution, onto the sensor coated with the five different examined PPFs, the maxima of the interference fringes are shifted in wavelength. This shift allows evaluating the optical thickness of the adsorbed BSA layer using a fast spectral correlation (FSC) method ${ }^{31}$ by assuming the refractive indices of BSA and PBS as 1.599 and 1.337 , respectively ${ }^{32,33}$.

The system set-up provides the passage of the solutions over the PPF-coated TInAS sensors mounted in a small fluid cell at $20 \pm 0.05{ }^{\circ} \mathrm{C}$. Prior to injection of $2 \mu \mathrm{L}$ of BSA solution ( $5 \mathrm{mg}$. $\mathrm{mL}^{-1}$ in PBS) into the cell, the PPF-coated sensor surfaces were exposed to pure PBS for 16 hours ensuring the complete hydration, which led to the acquisition of a flat baseline. With the assumption of the refractive indices of polymer films

$\mathrm{SiOx}=1.48, \mathrm{SiO}: \mathrm{CH}=1.55$ and for the gradients 1.50$)^{34-36}$, the BSA adsorption isotherm was monitored with TInAS over $65 \mathrm{~min}$ and the nominal thickness of the adsorbed layer in nanometers was determined. These thickness $(D)$ values can be converted to the adsorbed mass per surface area $\left(\mathrm{ng} \mathrm{cm}^{-2}\right)$ using the equation ${ }^{29}$ :

$$
M=\frac{n_{A}-n_{S}}{d n / d c} \times D
$$

which takes the dry refractive index of the adsorbate $n_{A}$, the solvent refractive index $n_{S}$, and the refractive index increment $d n / d c$ into account. A refractive index increment value $d n / d c=0.182$ $\mathrm{cm}^{3} / \mathrm{g}$ was used, besides the above mentioned BSA (adsorbate) and PBS (solvent) refractive index 6.

\section{Results and Discussion}

The plasma polymerization process used here converts a molecule in the gas phase, the "monomer", via electron-impact activation and dissociation into a reactive film-forming species. After arrival on the substrate, the film forming species forms a deposit, the plasma polymer film (PPF). While the plasma environment is far from thermodynamic equilibrium, a wide variety of reactions can occur depending on the electron energy distribution function. Particularly, the wellstudied monomer, hexamethyldisiloxane (HMDSO), shows a rather defined reaction pathway via formation of $\left(\mathrm{CH}_{3}\right)_{2}$-Si-O radicals having a sticking probability close to one ${ }^{10,37}$. Thus, high deposition rates are typically observed (up to several $\mu \mathrm{m} \mathrm{min}^{-1}$ ) ${ }^{38,39}$, which are proportional to the monomer gas flow rate per deposition area ${ }^{37}$. In order to better control the deposition of vertical depth-gradients, namely including the cover layers at the nanoscale, the deposition rates were thus 
lowered to about $25 \mathrm{~nm} \mathrm{~min}^{-1}$ by using a comparably large electrode (deposition) area of $1500 \mathrm{~cm}^{2}$ combined with a low HMDSO flow rate of $4 \mathrm{sccm}$. To obtain a stable homogeneous plasma, Ar was added at $20 \mathrm{sccm}$ at a low pressure of $7 \mathrm{~Pa}$. Thus, plasma-polymerized, hydrophobic $\mathrm{SiO}: \mathrm{CH}$ cover films were achieved; they retained a high amount of $\mathrm{CH}_{3}$ groups at the input power of 50 W. Addition of $\mathrm{O}_{2}$ at $40 \mathrm{sccm}$ enabled to further oxidize the hydrocarbon portion in the growing films, yielding inorganic SiOx films. Namely, a power input of $100 \mathrm{~W}$ and an $\mathrm{O}_{2}: \mathrm{HMDSO}$ ratio of 10:1 were selected to obtain a nanoporous film structure exhibiting moderately hydrophilic properties ${ }^{10}$. It was noted that higher gas ratios and power input lead to increasing ion bombardment during deposition, which would result in the formation of more dense barrier coatings limiting the water penetration potential ${ }^{40}$.

Using these two selected plasma conditions, the two-step depositions were realized by deposition of a $50 \mathrm{~nm}$ SiOx base layer (1), followed by a nominally $4 \mathrm{~nm}$ thick SiO:CH cover layer (2); due to the different conditions and the implantation depth of reactive species, a nanometer wide hydrophilic-to-hydrophobic transition is inevitably formed in the subsurface (named Vgrad) as described in Fig. 1. Indeed, the gradient morphology underneath the surface is the result of relaxation of unsaturated bonds relaxing on the time scale of hours ${ }^{41}$. Optionally, an intermediate oxidizing plasma step was introduced between layer (1) and layer (2), mainly focusing on 1 or 5 minutes duration (1* and $1^{* *}$, named Vgrad-ox1 and Vgrad-ox5, respectively). This optional oxidation within the in-situ multi-step process was realized by closing the HMDSO supply at the end of the base layer deposition, yielding enriched oxygen and depleted hydrocarbons at the surface before the cover layer deposition step was initiated. The resulting variety of siloxane functional vertical gradient films were stable and systematically studied. A goal of this study was to quantify water intrusion into the various graduated plasma-polymer matrix, relating to the hypothesis that this water can be in a different state/orientation rather than in a bulk state configuration, giving rise to dipole field effects that can range $>20 \mathrm{~nm}$ from the subsurface to affect the adsorption of (dipolar) proteins near the surface ${ }^{6}$. For this purpose, the different plasma polymerized vertical gradient films, as well as several controls consisting of $\mathrm{SiOx}$ and $\mathrm{SiO}: \mathrm{CH}$ plasma polymers, were assessed with respect to their surface and subsurface chemical structure as well as their hydration characteristics. These well-characterized and hydrated PPFs were then used to probe protein-surface interactions resulting in an extended range of adsorbed protein thickness as depicted in Fig. 1. The material properties enabling this additional control of protein adsorption considering equal surface characteristics are further discussed in the following sections. 


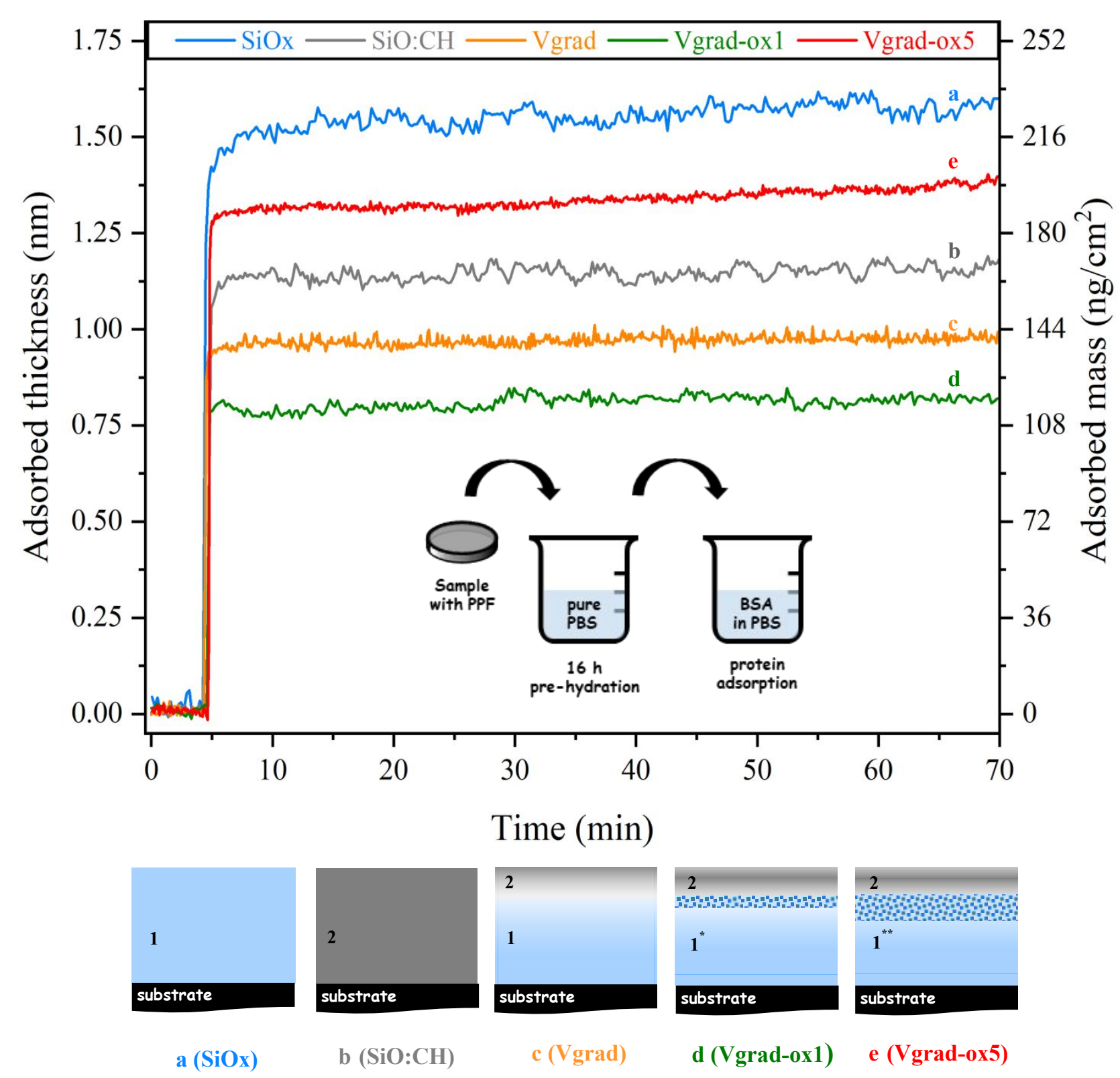

Figure 1. Optical thickness of Bovine Serum Albumin (BSA) adsorbed onto subsurface hydrated gradient plasma polymer films (Vgrad - orange curve, Vgrad-ox1 - green curve, and Vgrad-ox5 - red curve). The measured optical thicknesses were transformed into the adsorbed mass per area, which is displayed on a second y-axis. Siloxane functional PPFs with a vertical gradient embedded into the subsurface are schematically illustrated below the figure; Vgrad (c), Vgrad-ox1 (d) and Vgrad-ox5 (e). Layer (1) describes a hydrophilic SiOx base layer while Layer $\left(1^{*}\right)$ and $\left(1^{* *}\right)$ indicate the 1 and 5 minutes plasma oxidized hydrophilic SiOx base layers, respectively, prior to the deposition of the hydrophobic SiO: $\mathrm{CH}$ cover layer (2). Adsorption on the hydrophilic base layer ( $\mathrm{SiOx}$ - light blue curve (a)) and on the hydrophobic top layer ( $\mathrm{SiO}: \mathrm{CH}$ - gray curve (b)) are shown for reference. During the time-resolved protein adsorption experiment, BSA solution ( $5 \mathrm{mg}$ $\mathrm{mL}^{-1}$ in PBS) was injected after the pre-immersion of PPFs in pure PBS over $16 \mathrm{~h}$. The adsorption curves here are exemplary. 


\section{Plasma discharge analyses}

The optical emission spectrum (OES) of the plasmas fed by Ar/HMDSO (20:4) gas and $\mathrm{Ar} / \mathrm{HMDSO} / \mathrm{O}_{2}(20: 4: 40)$ gas at a pressure of $7 \mathrm{~Pa}$ and $\mathrm{RF}$ power of $50 \mathrm{~W}$ and $100 \mathrm{~W}$, respectively, are compared in Fig. 2, reporting the main emitted lines in accordance with literature data $36,42,43$. Increase of power input from $50 \mathrm{~W}$ to $100 \mathrm{~W}$ was needed to account for excitation (dissociation) of oxygen in order to remove hydrocarbons and thus form a denser, inorganic SiOx film ${ }^{10}$. As expected, the gas phase analysis of Ar/HMDSO plasma with (Fig. 2b) or without oxygen dilution (Fig. 2a), supported the generation of inorganic and polymer-like coatings, respectively. Herein, relevant species in the OES spectrum such as $\mathrm{O}, \mathrm{H}$, and $\mathrm{CO}$ were selected for a further detailed discussion, since they play a crucial role in the deposition of siloxane coatings. Note that the emission from the Ar carrier gas, namely Ar I and Ar II intensities were found to be comparable for both gas compositions despite the use of a higher power in the cases with $\mathrm{O}_{2}$ added in the plasma. This invariance is likely due to lowered electron densities by addition of oxygen that consumes energy in the plasma. Most of all, a significant difference between the intensity of the emission lines from atomic $\mathrm{O}(777 \mathrm{~nm})$ and the Balmer $\mathrm{H}$ lines $\left(\mathrm{H}_{\alpha}\right.$ and $\mathrm{H}_{\beta}$ at 656 and $486 \mathrm{~nm}$, respectively) can be noticed in Fig. $2 a$ and $2 b$. The admixture of oxygen at the ratio of 10:1 with respect to HMDSO left a detectable amount of (excess) atomic $\mathrm{O}$ formed in the plasma indicative of distinct oxidizing conditions during film growth ${ }^{44}$. Accordingly, the $\mathrm{C}_{2}$ peaks completely disappeared, while the prominent CO lines appeared. This can be interpreted as hydrocarbon removal from the $\mathrm{PPF}$, which consumes atomic $\mathrm{O}$ and forms volatile $\mathrm{CO}$ and $\mathrm{CO}_{2}$ while depositing inorganic SiOx. Along the same lines, an increase in $\mathrm{H}$ concentration in the more oxygen-rich plasma can be understood as dehydrogenation of the surface by etching with atomic $\mathrm{O}{ }^{45}$, which agrees with previous analyses of the chemical film composition ${ }^{25}$. 

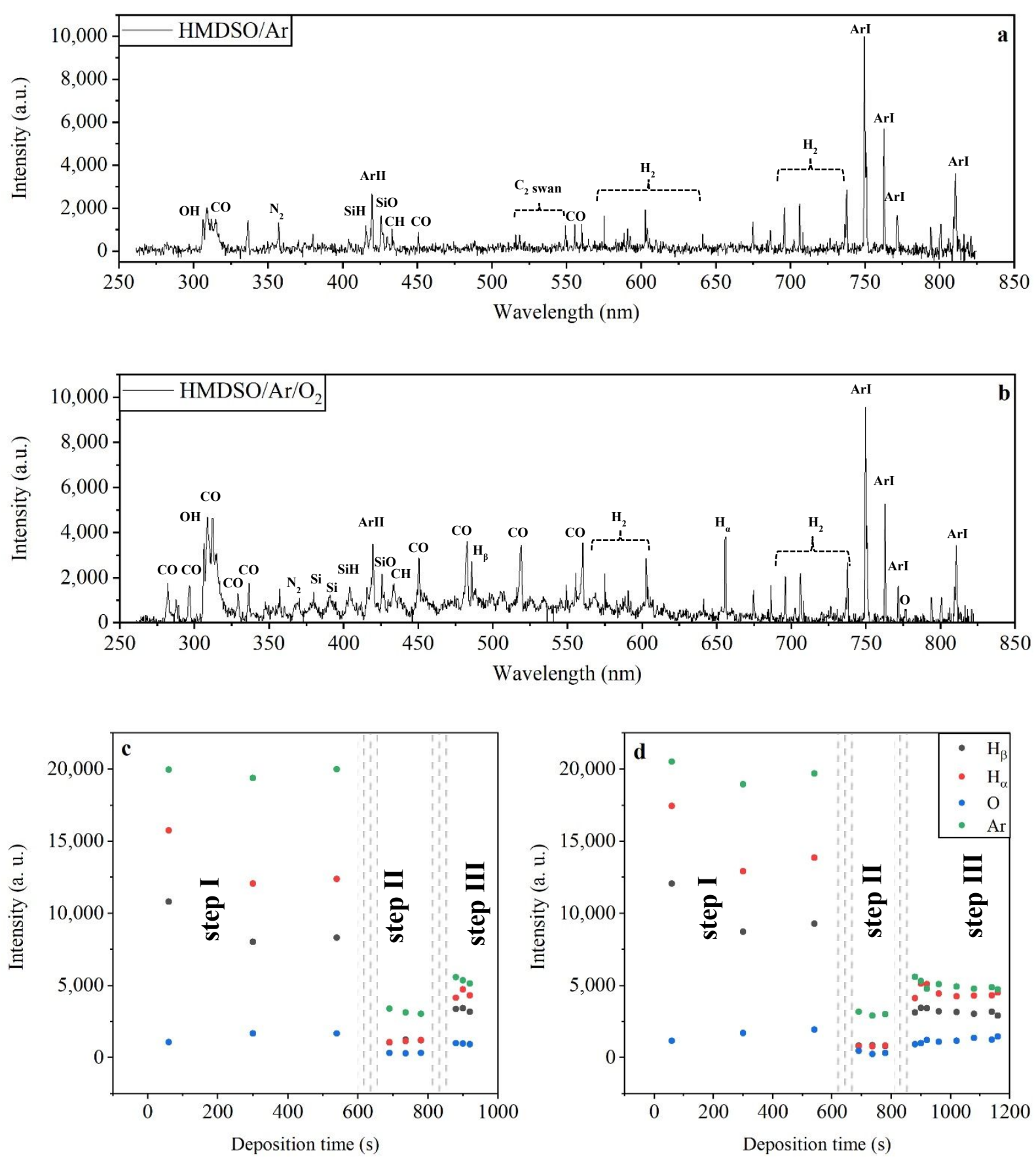

Figure 2. Plasma discharge analysis evaluated by optical emission spectroscopy (OES). Intensities of emission lines of various species obtained in Ar/HMDSO (20:4) plasmas observed at $7 \mathrm{~Pa}$, either a) without oxygen at the power of $50 \mathrm{~W}$ or b) with added $40 \mathrm{sccm} \mathrm{O}_{2}$ flow at $100 \mathrm{~W}$. Evolution of $\mathrm{Ar}, \mathrm{H}$ and $\mathrm{O}$ emissions during the deposition of c) Vgrad-ox1 and d) Vgrad-ox5 observed by OES (step I: $\mathrm{Ar} / \mathrm{O}_{2}$ pretreatment for $10 \mathrm{~min}$, step II: $\mathrm{SiOx}$ deposition for $2.5 \mathrm{~min}$, step III: plasma oxidation for 1 and $5 \mathrm{~min}$, respectively). The areas indicated by gray dashed lines correspond to plasma-off time during the transition between the different processes.

The intensities of $\mathrm{H}_{\alpha}, \mathrm{H}_{\beta}$, Ar and $\mathrm{O}$ emission lines observed during the deposition of Vgrad-ox1 and Vgrad-ox5 films are compared in Figures 2c and 2d. As it was described in the experimental part, the film deposition of Vgrad-ox1 and Vgrad-ox5 included the following steps; step I: a 
substrate pre-cleaning with $\mathrm{Ar} / \mathrm{O}_{2}(80 / 20 \mathrm{sccm})$ plasma at $200 \mathrm{~W}$; step II: deposition of $50 \mathrm{~nm}$ $\mathrm{SiOx}$ base layer with $\mathrm{Ar} / \mathrm{O}_{2} / \mathrm{HMDSO}(20 / 40 / 4 \mathrm{sccm})$ plasma at $100 \mathrm{~W}$; step III: $\mathrm{Ar} / \mathrm{O}_{2}(20 / 40$ sccm) plasma oxidation at $100 \mathrm{~W}$ for $1 \mathrm{~min}$ or $5 \mathrm{~min}$, and, step IV: generation of a $4 \mathrm{~nm} \mathrm{SiO}: \mathrm{CH}$ cover layer with Ar /HMDSO (20/4 sccm) plasma at $50 \mathrm{~W}$. Between each step, approximately 12 minutes plasma-off phase was introduced to allow for gas exchange. Since $\mathrm{H}_{\alpha}, \mathrm{H}_{\beta}$, and $\mathrm{O}$ emission lines were not present in step IV, the presented OES evolution of the multi-step process encompasses only steps I-III in Figure 2 . In accordance with the literature ${ }^{36,46}$, the Ar line intensity was increased during the pre-cleaning process as compared to steps II and III, due to a higher power resulting in an enhanced electron density. The observed decrease in the intensity of the Ar line during step II, as compared to step III, can be attributed to the HMDSO monomer addition, since part of the electron energy is consumed for HMDSO dissociation yielding a lower electron density ${ }^{47}$. Additionally, pronounced hydrocarbon etching from the deposited film by atomic oxygen can be observed in step III as indicated by the increase in $\mathrm{H}$ and $\mathrm{O}$ lines, which was as further prolonged for Vgrad-ox5.

\section{Surface characterization of the plasma polymer films}

The obtained films exhibited variations in the composition of the subsurface relative to the reference single-layer films in the form of vertical chemical gradients. All films were thus characterized for water wettability, the topography of the film surfaces and near-surface chemical composition by contact angle measurement (WCA), atomic force microscopy (AFM) and X-ray photoelectron spectroscopy (XPS), respectively, as reported in Table 1. Since all gradient films were terminated with the same nominally $4 \mathrm{~nm}$ thick SiO:CH cover layer, they are expected to exhibit the same water contact angle. The consistently observed water contact angle of $105^{\circ}$ indeed agrees with the $\mathrm{SiO}: \mathrm{CH}$ single-layer reference indicating the cover layer to be homogenous providing full coverage ${ }^{5}$. To exclude an influence of surface roughness on the observed WCA ${ }^{48}$, the root mean square roughness, $\mathrm{R}_{\mathrm{rms}}$, (over different areas of $5 \times 5 \mathrm{~m}^{2}$ ) of all surfaces was assessed by AFM, showing almost identical smooth surfaces with a roughness around $0.3 \mathrm{~nm}$ (see Table 1 and Supporting Information for further details). It is noted that the WCA measurement is a very surface sensitive technique, probing only the surface energy of the top surface with a depth sensitivity comparable to the surface roughness ${ }^{49}$. Additional characterizations are required to assess subsurface variations of the film properties. To characterize deeper layers, PPFs without cover layer were also prepared and examined. A lower, moderate hydrophilic water contact angle of $72^{\circ}$ was detected on the reference $\mathrm{SiOx}$ surface, whereas after the optional plasma oxidation 
step, significantly more hydrophilic surfaces were found, namely with a WCA of $\sim 20^{\circ}$ after 1 min oxidation and complete wetting after 5 min oxidation.

Table 1. Properties of siloxane plasma polymer films with different layer sequences

\begin{tabular}{|c|c|c|c|c|c|c|}
\hline Film & Layer sequence & WCA (deg.) & $\mathbf{R}_{\mathrm{rms}}(\mathbf{n m})$ & $\mathbf{C} / \mathbf{S i}$ & $\mathbf{O} / \mathbf{S i}$ & $\begin{array}{r}\text { Density } \\
\left(\mathrm{g} / \mathrm{cm}^{3}\right)\end{array}$ \\
\hline SiOx & (1) & 72 & 0.22 & 0.40 & 2.0 & 1.9 \\
\hline $\mathrm{SiO} x+1 \min o x$ & $\left(1^{*}\right)$ & 20 & 0.32 & 0.18 & 2.2 & 1.8 \\
\hline $\mathrm{SiOx}+5 \min \mathrm{ox}$ & $\left(1^{* *}\right)$ & $<5$ & 0.38 & 0.14 & 2.2 & 1.7 \\
\hline SiO:CH & (2) & 105 & 0.31 & 2.0 & 0.9 & 1.25 \\
\hline Vgrad & $(1)+(2)$ & 105 & 0.29 & 1.3 & 1.1 & - \\
\hline Vgrad-ox1 & $\left(1^{*}\right)+(2)$ & 105 & 0.32 & 1.0 & 1.3 & - \\
\hline Vgrad-ox5 & $\left(1^{* *}\right)+(2)$ & 105 & 0.29 & 1.1 & 1.2 & - \\
\hline
\end{tabular}

The chemical composition of all aforementioned PPFs was analyzed by XPS using high-resolution detail spectra obtained at a take-off angle of $45^{\circ}$, as reported in Table 1 . The results are in agreement with previous studies on HMDSO-based plasma polymers 10,17, 22, 50. As expected, the SiO:CH film showed a PDMS-like chemical composition with a $\mathrm{C} / \mathrm{Si}$ ratio of 2 and an O/Si ratio of $0.9^{50}$. The presence of oxygen in the $\mathrm{Ar} / \mathrm{O}_{2} / \mathrm{HMDSO}$ plasma increased the $\mathrm{O} / \mathrm{Si}$ ratio to 2, while decreasing the $\mathrm{C} / \mathrm{Si}$ ratio to 0.4 , as expected for the more inorganic $\mathrm{SiOx}$ film, yet retaining a small concentration of (hydro-)carbon. The elemental ratios of the reference layers correlate with the WCA values measured at the surfaces ${ }^{10,17}$. Due to the information depth of XPS (few nanometers), the gradient films revealed intermediate values of $\mathrm{O} / \mathrm{Si}$ and $\mathrm{C} / \mathrm{Si}$ ratios indicating sensitivity to the subsurface composition. The performed oxidation steps were found to further increase the measured $\mathrm{O} / \mathrm{Si}$ ratios while lowering the $\mathrm{C} / \mathrm{Si}$ ratios, which can be best seen by direct comparison of XPS signals with/without $\mathrm{SiO}: \mathrm{CH}$ cover layer. The variation in oxidation time (1 and $5 \mathrm{~min}$ ), 
however, did not yield a further detectable difference in $\mathrm{O} / \mathrm{Si}$ of those films using XPS. Thus, for 0.5 and 3 min plasma oxidation, similar properties can be expected.
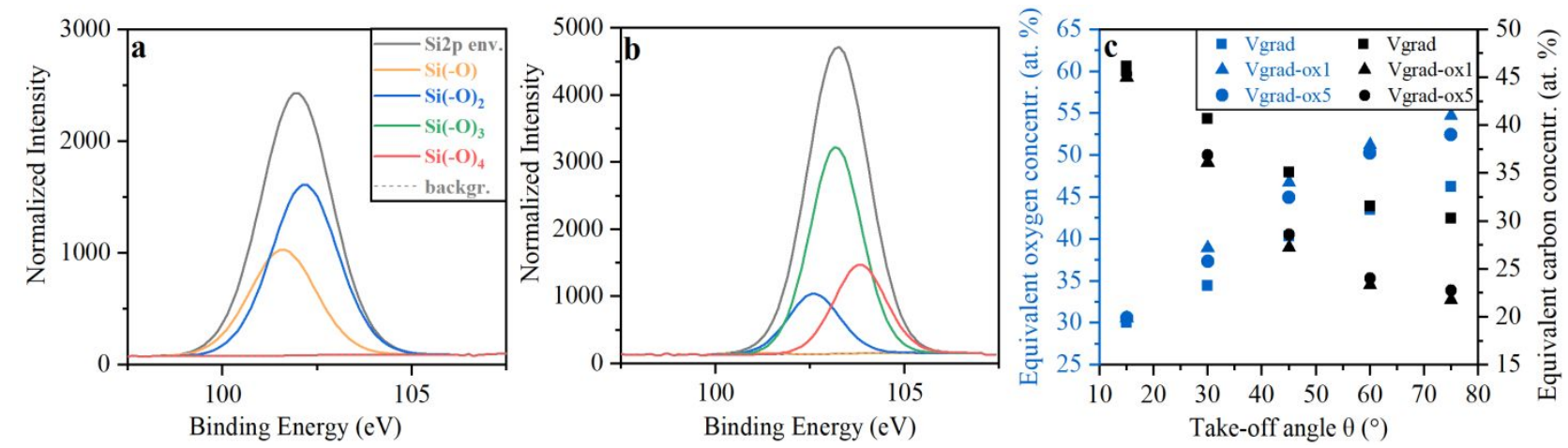

Figure 3. Chemical composition analysis by X-Ray Photoelectron Spectroscopy (XPS). XPS Si2p detail spectra deconvolution for single-layer references; a) $\mathrm{SiO}: \mathrm{CH}$ and b) $\mathrm{SiOx}$ films. The bold gray line represents the experimental Si $2 \mathrm{p}$ envelope and the orange, light blue, green and red lines represent the energy-fixed deconvolution into four possible oxidation states of $\mathrm{Si}$ atom $(\mathrm{Si}(-\mathrm{O})$, $\mathrm{Si}(-\mathrm{O})_{2}, \mathrm{Si}(-\mathrm{O})_{3}$, and $\mathrm{Si}(-\mathrm{O})_{4}$ respectively) from the curve fitting of the high-resolution $\mathrm{Si} 2 \mathrm{p}$ spectra. (c) The angular dependence of the oxygen (left axis) and carbon (right axis) concentration (homogenous film equivalents) for the gradient PPFs: Vgrad, Vgrad-ox1, and Vgrad-ox5 are represented as square, triangle, and circle, respectively, where light blue indicates the $\mathrm{O}$ content and black the $\mathrm{C}$ content.

Furthermore, the Si 2 p core level was fitted using four different oxidation states of the Si atom as described in the experimental section. Figure $3 \mathrm{a}$ and $3 \mathrm{~b}$ depict the observed curve fittings for the reference SiO:CH and SiOx films clearly showing their different compositions. The Ar/HMDSO film (Fig. 3a) consists of $\mathrm{Si}(-\mathrm{O})(34 \%)$ and $\mathrm{Si}(-\mathrm{O})_{2}(66 \%)$ oxidation states. This finding is in agreement with the retention of hydrocarbons and also indicates a less cross-linked, PDMS-like network, which is in a good agreement with the literature ${ }^{45}$. On the other hand, with the admixture of oxygen to the Ar/HMDSO plasma, $\mathrm{Si}(-\mathrm{O})$ oxidation states were no longer observed. Rather, the main constituents are now associated with $\mathrm{Si}(-\mathrm{O})_{2}(17 \%), \mathrm{Si}(-\mathrm{O})_{3}(58 \%)$ and $\mathrm{Si}(-\mathrm{O})_{4}(25 \%)$, all in agreement with a more cross-linked, $\mathrm{SiO}_{2}$-like film structure. Additionally, plasma oxidation of the SiOx reference films for $1 \mathrm{~min}$ and $5 \mathrm{~min}$ resulted in different oxidation state compositions. While the fitting of $\mathrm{Si} 2 \mathrm{p}$ peak of 1 min oxidized SiOx film mainly consists of $\mathrm{Si}(-\mathrm{O})_{2}$ and $\mathrm{Si}(-\mathrm{O})_{3}$ oxidation states, $\mathrm{Si}(-\mathrm{O})_{3}$ and $\mathrm{Si}(-\mathrm{O})_{4}$ oxidation states predominantly exist in the 5 min oxidized SiOx sample. These differences in oxidation state are expected to affect the subsurface region inside the Vgrad-ox1 and Vgrad-ox5 films.

Finally, film densities of the siloxane coatings were derived from measurements of the deposited gravimetric mass and edge thickness by profilometry; these density measurements were carried 
out using a defined area on glass slides. The relatively low densities determined for SiO: $\mathrm{CH}$ (1.25 $\left.\pm 0.02 \mathrm{~g} \mathrm{~cm}^{-3}\right)$ and $\mathrm{SiOx}\left(1.9 \pm 0.02 \mathrm{~g} \mathrm{~cm}^{-3}\right)$, agree with results of previous studies ${ }^{25}$ and imply a nanoporous structure. Note that $\mathrm{SiO}_{2}$-like films as used for barrier coatings show distinctly higher densities. To estimate the density of SiOx after plasma oxidation for 1 and $5 \mathrm{~min}$ we had to increase the thickness and mass of the oxidized film portion. Therefore, ten successive deposition/oxidation cycles were applied; each time $20 \mathrm{~nm} \mathrm{SiOx}$ was deposited, followed by 1 or $5 \mathrm{~min} \mathrm{Ar} / \mathrm{O}_{2}$ plasma oxidation. The average film density of such gradients suggests a further reduction in film density. Hence, the oxidation step effectively removed even more hydrocarbons leaving a highly nanoporous structure agreeing with reported findings on gradient $\mathrm{SiO}: \mathrm{CH}$ coatings exhibiting low dielectric constant ${ }^{51}$.

\section{In-depth film characterization by AR-XPS and ToF-SIMS}

Angle-resolved XPS measurements were performed on gradient PPFs to provide additional information about the subsurface chemical composition. Over five different take-off angles $\left(15^{\circ}\right.$, $30^{\circ}, 45^{\circ}, 60^{\circ}$, and $75^{\circ}$ ) the information depth was gradually varied from approximately 2 to $9 \mathrm{~nm}$ covering the hydrophilic-to-hydrophobic transition region. Figure $3 \mathrm{c}$ displays the oxygen and carbon concentrations (homogeneous film equivalent) for the three studied gradient coatings as a function of emission angle. As expected, the highest carbon content with the lowest oxygen concentration was obtained at small angles. Here, the signal originates from the outermost $\sim 2 \mathrm{~nm}$, which essentially reflects the $\mathrm{SiO}: \mathrm{CH}$ cover layer composition. With increasing depth information oxygen was found to increase at the expense of carbon, indicating the transition towards the inorganic SiOx base layer composition. While the surface composition was comparable for all gradient PPFs, the two films with additionally oxidized interface demonstrated a noticeably enhanced $\mathrm{O}$ content in the subsurface region.

Since XPS signals exhibit an exponentially decreasing sensitivity with depth, there are some limits in the detection of subsurface variations in the chemical composition. To obtain complementary information about the depth profile, ToF-SIMS analysis was performed on the same reference and gradient films. As mentioned before, full depth profiles, obtained by sputtering the nominally 50 $\mathrm{nm}$ thick coatings; i.e. until reaching the Si substrate, are shown here. Figure 4 compares the indepth evolution of different fragments, $\mathrm{SiC}_{2} \mathrm{H}_{5} \mathrm{O}^{-}$(Fig. 4a), $\mathrm{SiOH}^{-}$(Fig. 4b) and $\mathrm{SiH}_{2} \mathrm{O}_{3}^{-}$(Fig. 4c), for all samples. The presence of the $\mathrm{SiC}_{2} \mathrm{H}_{5} \mathrm{O}^{-}$fragment displays PDMS-like film properties ${ }^{52,53}$, whereas the $\mathrm{SiH}_{2} \mathrm{O}_{3}{ }^{-}$fragment is specific for inorganic, $\mathrm{SiO}_{2}$-like film structures, also providing information on cross-linking ${ }^{54}$. On the other hand, the existence of $\mathrm{SiOH}^{-}$groups in the PPF is an indication for porosity as discussed earlier ${ }^{55-57}$. 
The average sputtering rate for ToF-SIMS was found to be higher (roughly doubled) for SiO: $\mathrm{CH}$ compared to $\mathrm{SiOx}$ by measurement of the sputtering time required to pass through the $50 \mathrm{~nm}$ thick reference coatings. The difference in sputtering rates for the two references can be referred to the different film characteristics such as density, cross-linking and organic/inorganic nature, which were confirmed by XPS and film density calculation. Varying sputtering conditions thus need to be considered to determine the transitions between the gradient PPFs. Hence, the depth profiles are presented with respect to sputtering time, which might not refer to the same depth for the different coatings. Furthermore, the sputtering rate is expected to be higher during the initial phase of the sputtering process; leading to a shift of the nominal depth scale towards the surface ${ }^{58}$. In addition, initial sputter-induced effects at the outermost region of the surface (transient region, $\sim 1$ $\mathrm{nm}$ ) does not allow an exact determination of the nominally $4 \mathrm{~nm}$ thick SiO:CH cover layer thickness, this non-linear initial sputtering phase was thus indicated by gray shaded area in Fig. $4 \mathrm{a}, 4 \mathrm{~b}$, and $4 \mathrm{c}$. Nevertheless, the gradient films showed a similar qualitative behavior of $\mathrm{SiC}_{2} \mathrm{H}_{5} \mathrm{O}^{-}$, $\mathrm{SiOH}^{-}$and $\mathrm{SiH}_{2} \mathrm{O}_{3}{ }^{-}$fragments in the near-surface region as compared to the reference $\mathrm{SiO}: \mathrm{CH}$ film, clearly indicating the full coverage by $\mathrm{SiO}: \mathrm{CH}$. The $\mathrm{SiC}_{2} \mathrm{H}_{5} \mathrm{O}^{-}$intensity, indicative for the polymerlike, hydrophobic cover layer, vanished within the first $\sim 25 \mathrm{~s}$ of sputtering, while the $\mathrm{SiH}_{2} \mathrm{O}_{3}{ }^{-}$ fragments increased to the level of the SiOx reference film over a slightly longer sputtering time. Likewise, the $\mathrm{SiOH}^{-}$counts dropped according to the raise in $\mathrm{SiH}_{2} \mathrm{O}_{3}{ }^{-}$fragments. The cover layer thus exhibits an increased oxygen content close to the $\mathrm{SiO}: \mathrm{CH} / \mathrm{SiOx}$ interface in agreement with the results from ARXPS, which might be caused by the plasma interaction during the initial deposition phase. Moreover, as depicted in Fig. 4, gradient films exhibit a gradual change in the counts of these fragments from the surface to the bulk region, confirming the existence of chemical vertical gradients. Although gradients are induced by the in-situ multistep plasma polymerization process, the interface of the gradient films might have undergone some post-oxidation processes, i.e. within hours after deposition due to radicals left in the film structure ${ }^{41,59}$, further supporting the formation of a vertical gradient defining the subsurface architecture. The variation in the vertical gradient structure by the intermediate plasma oxidation step might induce a difference in the width of the hydrophilic-to-hydrophobic transition zone. However, due to the mentioned difficulties to define a sputtering rate for the near-surface region as well as for the transition zone, no clear indication of the width can be given here ${ }^{60,61}$. As it is displayed in Fig. 4a, 4b, and 4c, we can only assess the middle of the transition zone, defining the x-position of the half height between min and max of the rising/falling part of the profile ${ }^{61}$. Hence, further analysis, e.g. Neutron Reflectometry, is required to quantify/qualify the difference between the transition widths of gradients. 
Finally, the abundance of silanol fragments in the profile of all siloxane PPFs confirmed the presence of unconnected network branches, i.e. akin to a nanoporous structure. The $\mathrm{SiOH}^{-}$ intensities were particularly pronounced in the $\mathrm{SiO}: \mathrm{CH}$ films as well as in the transition regions of the gradients films. This is in agreement with calculated lower densities of 1 and 5 minutes oxidized SiOx films ( $1^{*}$ and $1^{* *}$, respectively) (Table 1$)$. These $\mathrm{Si}-\mathrm{OH}$ groups also represent sites available for interaction with water molecules, allowing the reactive diffusion that is known to form diffusion-relevant channels of water intrusion ${ }^{62}$. Although at a reduced level for SiOx films, the silanol signal could be detected throughout the bulk composition of all layers, indicating the intended omnipresent openness of the Si-O network. Hence, a certain degree of hydration can be expected for all examined plasma polymer films. The deposition of a hydrophobic cover layer does not represent a complete diffusion barrier. We thus investigated hydration in more detail using Neutron Reflectometry (NR) in $\mathrm{D}_{2} \mathrm{O}^{25}$.
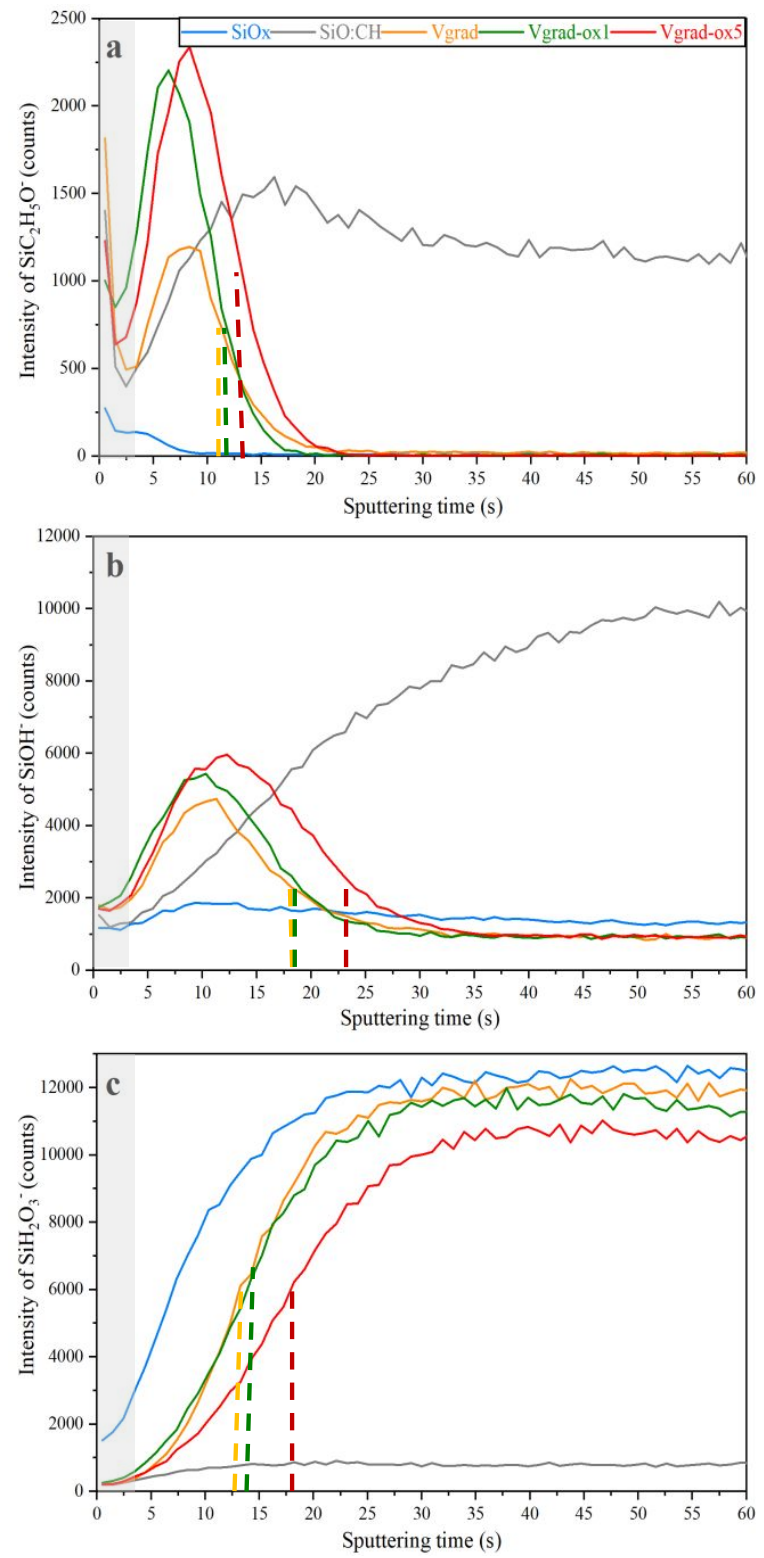
Figure 4. Time of flight secondary ion spectrometry (ToF-SIMS) depth profiles of characteristic film fragments of a) $\mathrm{SiC}_{2} \mathrm{H}_{5} \mathrm{O}^{-}$, b) $\mathrm{SiOH}^{-}$, and c) $\mathrm{SiH}_{2} \mathrm{O}_{3}{ }^{-}$for references ( $\mathrm{SiOx}$ - light blue, $\mathrm{SiO}: \mathrm{CH}$ - gray) and gradient PPFs (Vgrad - orange, Vgrad-ox1 - green, and Vgrad-ox5 - red). The gray shaded area in Fig. $4 a, 4 b$, and $4 c$ describe the transient region caused by ion beam. Transition middle of the gradients are marked by a dashed line with a corresponding curve color.

\section{Water penetration through gradient films}

The scattering length density (SLD) depth profiles of the two oxidized gradient plasma polymer films in the dry and hydrated states were assessed via NR measurements. The reflectivity curves for the gradient films using the intermediate 1 and 5 min plasma oxidation step on $\sim 50 \mathrm{~nm} \mathrm{SiOx}$ base layer obtained against air (dry state) and $\mathrm{D}_{2} \mathrm{O}$ (hydrated state) are shown in Figure 5a and 5c, respectively. The response of homogeneous plasma polymerized reference layers, $\mathrm{SiOx}$ and $\mathrm{SiO}: \mathrm{CH}$ to aqueous environments had been previously reported revealing water penetration for both films albeit at a slower rate with a lower water volume for the hydrophobic SiO:CH film ${ }^{25}$. While the NR data of the gradient films are inherently more complex, we can still assign certain spectral features to gradient structures. Interference of reflected neutrons at the polymer/air, polymer/ $\mathrm{D}_{2} \mathrm{O}$, and polymer/substrate interfaces results in the occurrence of Kiessig fringes; their periodicity indicates total film thickness and their amplitude depends on the SLD contrast at the film boundaries. It was previously reported that SiOx exhibits a higher SLD than SiO:CH, resulting in higher amplitudes of Kiessig fringes in the air ${ }^{25}$. We note that the presence of gradients with SLD contrast, including the oxidized gradient PPFs, typically results in the formation of two superimposed periodicities (e.g. higher amplitude and higher frequency for the thicker SiOx part and lower amplitude lower frequency for the thinner SiO: $\mathrm{CH}$ part).

The obtained specular reflection data were fitted to a gradient model of a film with variable SLD on the Si substrate. The dry state of Vgrad-ox1 and Vgrad-ox 5 films was fitted by 12 and 15 layers (excluding the native $\mathrm{SiO}_{2}$ layer) of variable thickness, density, and interface roughness. Not only the hydrophilic/hydrophobic gradient transition between the layers, but also the relatively thicker SiOx part of the films revealed a non-uniform density profile perpendicular to the surface. These findings are consistent with the results of the previous work obtained on the homogeneous singlelayer films ${ }^{25}$. The constructed density profiles for the oxidized gradients in the dry state originating from the best fits to the reflectivity data are presented in Figure $5 \mathrm{~b}$ and $5 \mathrm{~d}$ as gray solid lines (see Supporting Information for more details). Note that the two examined films were slightly different in their nominal total thicknesses which appeared in the presented SLD profiles. Similarly to PDMS, the SLD range of the hydrophobic cover layer ( $\mathrm{SiO}: \mathrm{CH})$ of the films was found to vary 
from $0.07 \times 10^{-6} \AA^{-2}$ to $0.3 \times 10^{-6} \AA^{-2}$, while the SLD further increased through the extended subsurface region, approaching the SLD value expected for the hydrophilic base layer ( $\mathrm{SiOx}$ ). Noticeably, the observed subsurface transition layer from $\mathrm{SiO}: \mathrm{CH}$ to $\mathrm{SiOx}$ properties, leading to a dramatic change in SLD, was observed to be $0.6 \mathrm{~nm}$ wider in the case of the longer plasma oxidation step. This result, which supports the existence of a transition zone and also quantifies its width, is complementary to the ToF-SIMS results.

To further study the hydration of gradient films, we incubated the films in $\mathrm{D}_{2} \mathrm{O}$ for 12 hours. The NR data of the hydrated Vgrad-ox1 film was modeled with 12 layers. Iterative manual fitting was allowing the variable thickness of selected layers, as well as density and interface roughness between multiple fitting steps. The obtained solution of this multi-variable fitting procedure is not unique, but some solutions can readily be excluded for lack of plausibility. Solutions with smallest $\chi^{2}$ value yielding reliable convergence and reasonable SLD profiles that were consistent with the dry state results were generally preferred. The number of layers required for a good fit had to be increased up to 19, in the case of the rather complex graduated and hydrated Vgrad-ox5 sample, in order to reach a satisfying agreement between the model fitting and the experimental reflectivity data (see Supporting Information for more details). In Figure $5 \mathrm{~b}$ and $5 \mathrm{~d}$, the best SLD profiles upon $\mathrm{D}_{2} \mathrm{O}$ exposure are presented for Vgrad-ox1 and Vgrad-ox5 as green solid lines. Most of all, the two oxidized gradient PPFs with varying oxidation time indicate a significant enhancement of $\mathrm{D}_{2} \mathrm{O}$ penetration through their graduated gradient film structure.

Our results confirm that water hydrates the gradient film, despite the hydrophobic character of the cover layer (SiO:CH). Due to the observed time-effects, hydration also seems to proceed in agreement with defect-driven (porous morphology) and concentration dependent-reactive diffusion models, which are principal water transport mechanisms in siloxane coatings as previously proposed ${ }^{63,64}$. The well-studied reactive diffusion mechanism describes the diffusion pathway of water molecules relating to the hydrolysis of Si-O-Si bonds and therefore the formation of silanol groups. Due to the difference in the concentration of Si-O-Si bonds, water intrusion in the hydrophobic porous film might proceed via single nanochannels. A more uniform hydration front is expected in the hydrophilic material ${ }^{65}$. Additionally, the transport of molecular water in siloxane films involves chemical reactions forming rather immobile $\mathrm{OH}$ groups ${ }^{63}$. Thus, the SiO-Si backbone in $\mathrm{SiO}: \mathrm{CH}$ films allows water migration, as supported by the $\mathrm{SiOH}$ content that was detected in the ToF-SIMs analysis ${ }^{10,25}$. As it was shown in Fig. 4b, there is an enrichment of silanol contents of gradients at their transition region. Once water molecules reach the buried hydrophilic/hydrophobic interfaces, the properties of the underlying layers determine the further course of hydration. The embedded plasma oxidation for $1 \mathrm{~min}$ thus resulted in a pronounced 
equilibrium accumulation of water molecules at the hydrophilic/hydrophobic interphase proceeding with an even front into the SiOx part of the film. The uniform increase in SLD over the entire hydrated $\mathrm{SiOx}$ bulk indicates the saturation might have been reached. The extended plasma oxidation step for $5 \mathrm{~min}$, which formed an enhanced concentration gradient and an even more porous interface, further facilitated diffusion yielding enhanced water accumulation along the gradient zone. This pronounced water uptake can be attributed to the significant increase in the formed $\mathrm{SiOH}^{-}$group content yielded by longer plasma oxidation of $\mathrm{SiOx}$ base layer ${ }^{66}$. Interestingly, the $\mathrm{SiO}: \mathrm{CH}$ cover layer overall remained essentially dry, which is indicative for separated water channels, while the porous more hydrophilic subsurface efficiently sucked water molecules through this finger-like water channels of the $\mathrm{SiO}: \mathrm{CH}$ layer. The amount of hydrogen bonding of water with its environment is affected by the matrix functionality as well as the overall water content ${ }^{65,67}$. From the obtained SLD differences in dry and hydrated state we can estimate that the $\mathrm{D}_{2} \mathrm{O}$ volume fraction in the hydrophobilic/hydrophobic interface of the Vgrad-ox5 PPF increased up to $\sim 80 \%$, while it was about $\sim 27 \%$ for Vgrad-ox1, which is already slightly higher than the $\mathrm{D}_{2} \mathrm{O}$ volume fraction observed for the $\mathrm{SiOx}$ reference ${ }^{25}$ (see Supporting Information for more details about estimation).
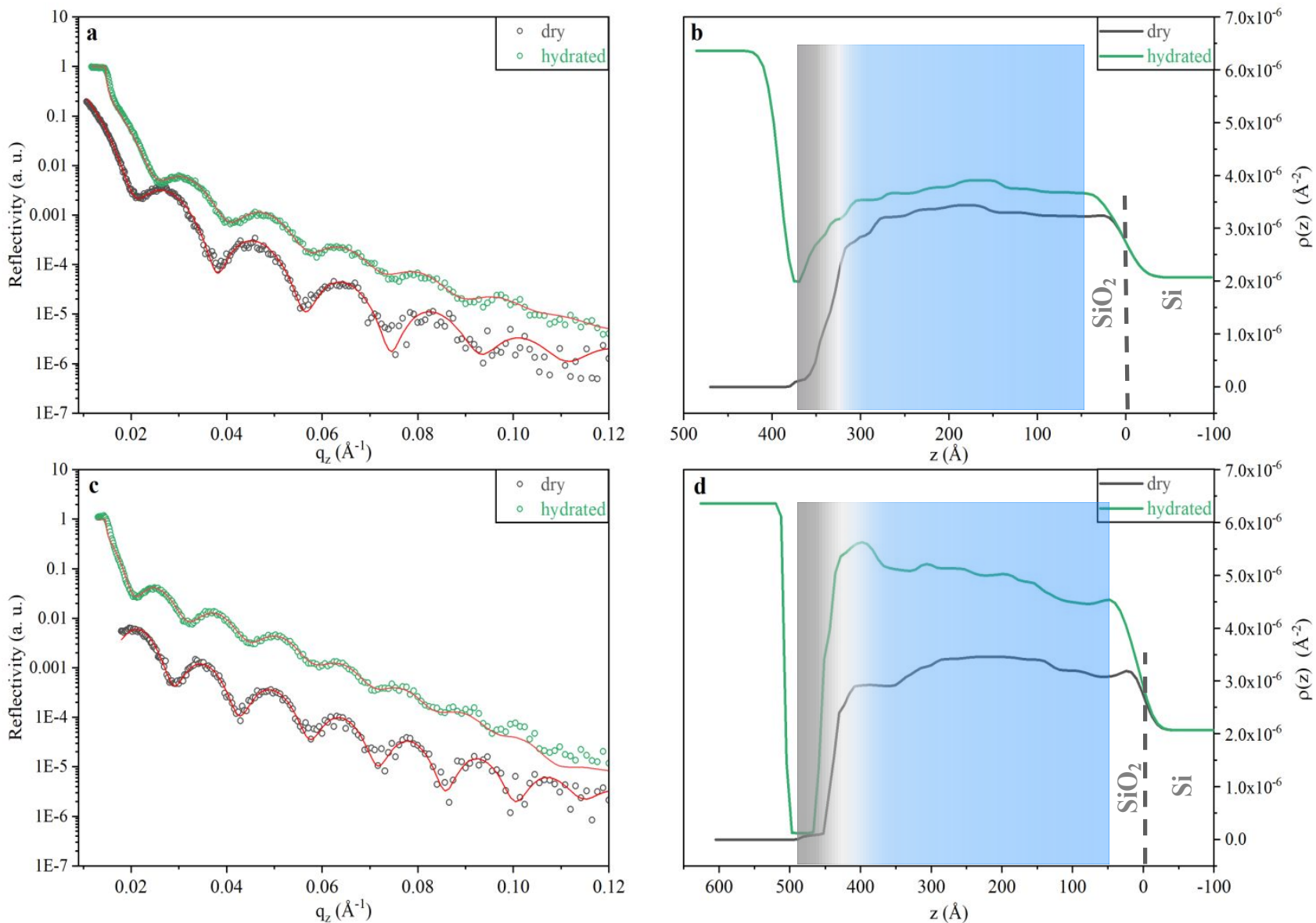

Figure 5. Specular neutron reflectivity (NR) curves and SLD profiles of gradient films; a-b) Vgrad-ox1 and c-d) Vgrad-ox5. The dry state (gray open circle symbol) and hydrated state (green 
open circle symbol) are shown. The red lines indicate the best SLD fits to the experimental data $\left(\chi^{2}=0.08\right.$ and 0.06 for dry states of Vgrad-ox 1 and Vgrad-ox 5, respectively, and 0.01 for hydrated states of the both films). Scattering length density (SLD) depth profiles of b) Vgrad-ox1 and d) Vgrad-ox 5 correspond to the best NR data fits shown on the left. The gray and light blue shaded areas represent the $\mathrm{SiO}: \mathrm{CH}$ and $\mathrm{SiOx}$ part of the films, respectively, while the pronounced gradient region from gray to light blue localizes the transition region between the two types of layers. The dark grey region corresponds to the location of the terminal cover layer.

\section{Subsurface water affects protein adsorption}

Water molecules can interact via hydrogen bonding, dipolar interactions, and van der Waals forces. Bulk water cannot align to yield a macroscopic dipole moment, since hydrogen bonding imposes a dominant tetragonal network that has no integral dipole moment. Inside a polymer matrix, hydrating water molecules are, on average, separated at a larger distance than in bulk water, which weakens the tetragonal hydrogen bonding order and thus unleashes a ferroelectric behavior. Dynamic dipolar nano-domains are thus expected to form and the range of dipolar forces $(>20 \mathrm{~nm})$ readily exceeds the one expected for hydrogen bonding and van der Waals $(<2 \mathrm{~nm})$. In the presence of chemical gradients in the polymer matrix, the average orientation of the dipoles may additionally be biased via water-matrix hydrogen bonding. The purpose of this section is to probe the effect of such long-range dipolar fields that are originating at the subsurface and range beyond the surface. Experimentally, we determine the equilibrium amount of adsorbed albumin protein on the surface. The strong permanent dipolar moment of albumin $\left(\mu_{\mathrm{BSA}}=384 \mathrm{D}\right){ }^{68}$ prompted us to use it as a model protein as it will be particularly sensitive to such dipolar fields.

The adsorption of BSA from buffer solution onto the different plasma polymer films was studied using TInAS, which is an interferometric method that detects changes in adsorbed film thickness 29. Figure 1 displays the time-resolved thickness of the adsorbed protein as measured by TInAS; here, the thickness is proportional to the adsorbed mass ${ }^{29}$. While Figure 1 shows exemplary adsorption curves for the examined PPFs, the average BSA adsorption amount as obtained from repeated measurements is displayed in Figure 6. In addition, the effect of the different intermediate plasma oxidation time (0-5 min) for the gradient structures is presented in comparison to the BSA adsorption range for $\mathrm{SiOx}$ and $\mathrm{SiO}: \mathrm{CH}$ reference films. We found that the adsorption of $\mathrm{BSA}$ molecules after $16 \mathrm{~h}$ hydration on the surface of the hydrophilic reference $\mathrm{SiOx}$ film reached the highest adsorbed mass of $1.6 \pm 0.04 \mathrm{~nm}$, while it was reduced to $1.1 \pm 0.08 \mathrm{~nm}$ on the hydrophobic reference $\mathrm{SiO}: \mathrm{CH}$ surface, as also reported in other studies ${ }^{5,69}$. These significant differences in adsorbed mass suggest that the protein-surface interaction, as well as the conformation of the 
adsorbed proteins, is different. Note that all samples had been immersed in PBS overnight prior to BSA addition to assure equilibrium hydration. In line with previous results ${ }^{5}$, a further reduced protein adsorption was observed on gradient films (Vgrad) with hydrophobic termination yielding $1.0 \pm 0.04 \mathrm{~nm}$. This previous study has also shown how these effects are directly linked to the hydration state of the gradient films. We now report that the BSA adsorption is even further suppressed on the 1 min plasma-oxidized gradient PPF (Vgrad-ox1), reaching the lowest adsorbed thickness of $0.87 \pm 0.03 \mathrm{~nm}$, as shown in Figure 6. It is further important to note that this effect is not proportional to oxidation time. While short oxidation for $0.5 \mathrm{~min}$ (Vgrad-ox0.5) gives comparable results to Vgrad, extending the oxidation time to 3 and $5 \mathrm{~min}$, on the contrary, led reproducibly to a noticeably increased adsorbed BSA mass around $1.3 \pm 0.1 \mathrm{~nm}$. Consider that all surfaces possessed a homogenous and flat surface topography (see Table 1) excluding the surface roughness impact on protein adsorption. Those results thus emphasize one more time the astonishingly strong sensitivity to the subsurface structure. As explored by NR, the oxidized gradients exhibited a significantly enhanced amount of embedded water molecules, particularly at the hydrophilic/hydrophobic interface. Referring to the hypothesis of ferroelectric water developed in previous studies 5,6 , the gradient architecture energetically supports the formation of matrixwater hydrogen bonding rather than the water-water hydrogen bonding as in bulk water, leading to the formation of ferroelectric water accumulating in the subsurface gradient region. Hence, these subsurface confined water molecules can generate dipole domains enabling long-range ( $>20 \mathrm{~nm})$ interaction forces with dipolar albumin adsorbing at the surface. The further reduced adsorbed mass of BSA molecules on 1 min oxidized gradient is remarkable and indicates an enhancement of the effect. The enhancement could be due to the increased concentration of oriented water molecules in the subsurface. A continued increase of water concentration in the gradient region will eventually re-establish the water-water hydrogen bonding suppressing its ferroelectric property. Indeed, the longer oxidation time ( 5 min plasma oxidation), which results in even higher water concentrations at the gradient (Fig. 5) eventually loses its capability of displaying dipolar domains and the amount of adsorbed protein increases again.

Hence, an optimum amount of water at the interface (quantity) corresponds to the lowering of BSA adsorption, which is referred to the potential orientation of water molecules (quality), whereas increasing water filling yields rather bulk water properties. 


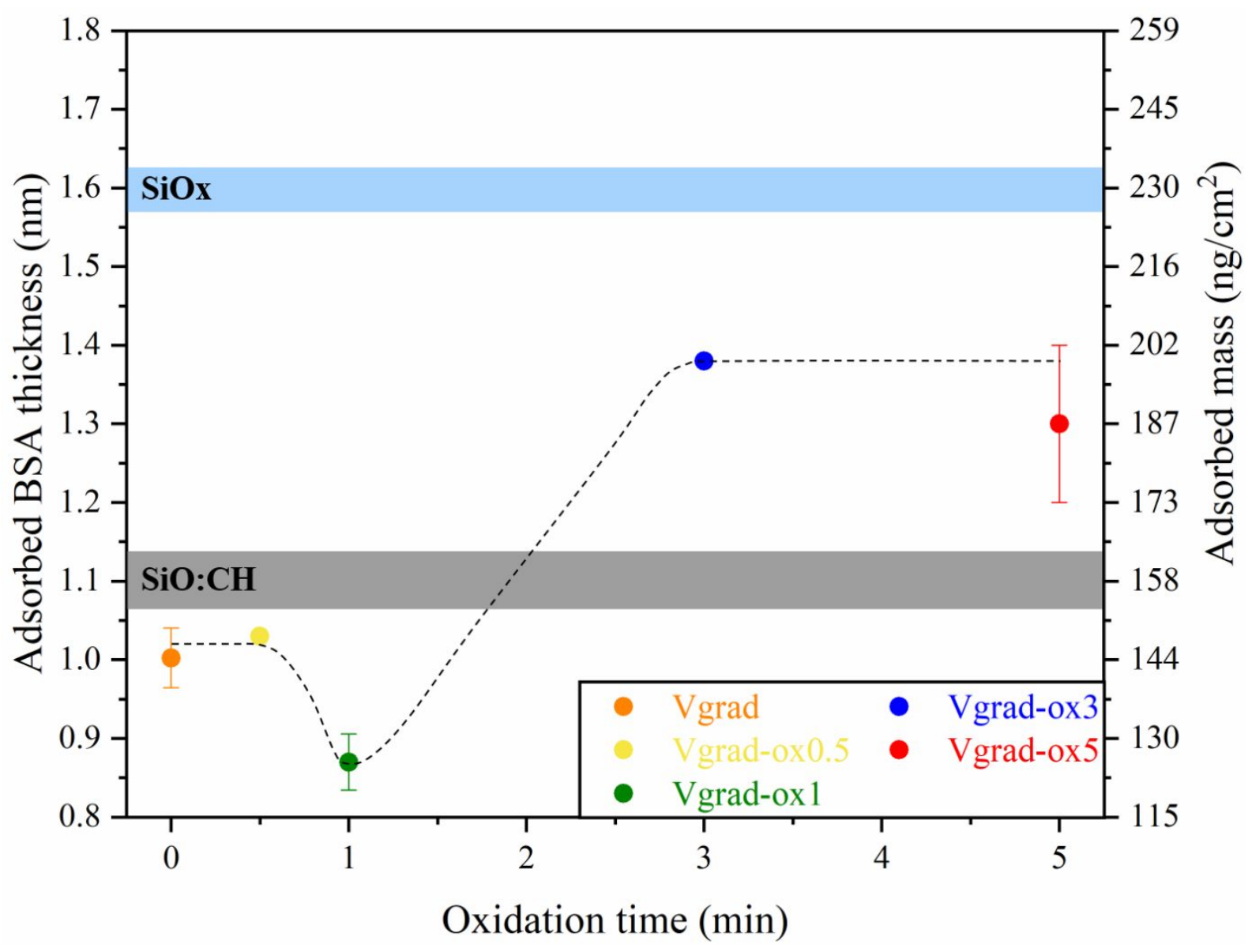

Figure 6. The effect of subsurface variation via plasma oxidation on adsorbed BSA amount. Plasma oxidation was applied on the SiOx base layer of gradient PPFs for 0, 0.5, 1, 3, and 5 min prior to deposit the $4 \mathrm{~nm}$ hydrophobic cover layer. As-deposited films were named as Vgrad (orange circle), Vgrad-ox0.5 (yellow circle), Vgrad-ox1 (green circle), Vgrad-ox3 (blue), and Vgrad-ox5 (red circle), respectively. The adsorbed BSA thickness on plain layers e.g. hydrophilic SiOx (light blue band) and hydrophobic SiO:CH (gray band) plasma polymer films are shown for reference. For better statistics, this figure includes data from several experiments, averaging the adsorption curve in the time interval from 20 to $70 \mathrm{~min}$.

\section{Conclusion}

An additional in-situ plasma oxidation step was introduced to modify previously established vertical chemical gradients as produced by an in-situ two step plasma polymerization process. This additional oxidation step altered the width and strength of the vertical chemical gradient, also yielding enhanced porosity as confirmed by AR-XPS, ToF-SIMS, and NR. NR measurementbased quantifications also showed that an extension of oxidation time from $1 \mathrm{~min}$ to $5 \mathrm{~min}$ increased the equilibrium water content from $\sim 27 \%$ to $\sim 80 \%$. The previously noted effect of reduced amounts of adsorbed albumin was confirmed; indeed, the adsorbed amount exhibits a 
minimum for plasma oxidation around $1 \mathrm{~min}$. This suggests that oxidation can intentionally be used to further reduce the protein adsorption by amplifying the subsurface effect. The subsequent vanishing of the effect at longer oxidation times is in agreement with the hypothesis involving different states of water molecules, e.g. ferroelectric water, because the observed significant increase of water content should inhibit any ferroelectric behavior of water molecules in favor of an increase of tetragonal inter-water hydrogen bonding.

In view of biomaterials applications, the introduction of the oxidation step effectively allows to extend the range of control of initial and fast albumin adsorption via subsurface design. As an outlook, this is particularly interesting since the presented effect should be particularly strong for dipolar proteins.

\title{
Associated Content \\ Supporting Information
}

Surface topography of plasma polymer films, Neutron reflectivity data fitting, and water content calculation (diffusion model).

\author{
Author Information \\ Corresponding author: \\ *E-mail: dirk.hegemann@empa.ch
}

\section{ORCID}

Ezgi Bülbül: 0000-0002-8777-357X

Patrick Rupper: 0000-0001-5513-512X

Thomas Geue: 0000-0002-7932-5574

Laetitia Bernard: 0000-0002-1965-7534

Manfred Heuberger: 0000-0001-5799-3785

Dirk Hegemann: 0000-0003-4226-9326

Acknowledgement: We gratefully acknowledge the financial support by the Swiss National Science Foundation (Project No. 200021_169180/1). This work is based on experiments performed at the Swiss spallation neutron source SINQ, Paul Scherrer Institute, Villigen PSI, Switzerland. 


\section{References}

1. Morgenthaler, S.; Zink, C.; Spencer, N. D., Surface-Chemical and-Morphological Gradients. Soft Matter 2008, 4 (3), 419-434.

2. Kim, M. S.; Khang, G.; Lee, H. B., Gradient Polymer Surfaces for Biomedical Applications. Progress in Polymer Science 2008, 33 (1), 138-164.

3. Elwing, H.; Gölander, C.-G., Protein and Detergent Interaction Phenomena on Solid Surfaces with Gradients in Chemical Composition. Advances in Colloid and Interface Science 1990, 32 (4), 317-339.

4. Ruardy, T.; Schakenraad, J.; Van der Mei, H.; Busscher, H., Preparation and Characterization of Chemical Gradient Surfaces and Their Application for the Study of Cellular Interaction Phenomena. Surface Science Reports 1997, 29 (1), 3-30.

5. Hegemann, D.; Blanchard, N. E.; Heuberger, M., Reduced Protein Adsorption on Plasma Polymer Films Comprising Hydrophobic/Hydrophilic Vertical Chemical Gradients. Plasma Processes and Polymers 2016, 13 (5), 494-498.

6. Hegemann, D.; Hocquard, N.; Heuberger, M., Nanoconfined Water Can Orient and Cause Long-Range Dipolar Interactions with Biomolecules. Scientific Reports 2017, 7 (1), 17852.

7. Rupper, P.; Vandenbossche, M.; Bernard, L.; Hegemann, D.; Heuberger, M., Composition and Stability of Plasma Polymer Films Exhibiting Vertical Chemical Gradients. Langmuir 2017, 33 (9), 2340-2352.

8. Vandenbossche, M.; Gunkel-Grabole, G.; Car, A.; Bernard, L.; Rupper, P.; ManiuraWeber, K.; Heuberger, M.; Faccio, G.; Hegemann, D., Near-Surface Structure of Plasma Polymer Films Affects Surface Behavior in Water and Its Interaction with Proteins. Plasma Chemistry and Plasma Processing 2018, 38 (4), 851-870.

9. Hegemann, D.; Körner, E.; Blanchard, N.; Drabik, M.; Guimond, S., Densification of Functional Plasma Polymers by Momentum Transfer During Film Growth. Applied Physics Letters 2012, 101 (21), 211603.

10. Blanchard, N. E.; Hanselmann, B.; Drosten, J.; Heuberger, M.; Hegemann, D., Densification and Hydration of Hmdso Plasma Polymers. Plasma Processes and Polymers 2015, 12 (1), 32-41.

11. Hegemann, D.; Hossain, M. M.; Körner, E.; Balazs, D. J., Macroscopic Description of Plasma Polymerization. Plasma Processes and Polymers 2007, 4 (3), 229-238. 
12. Hegemann, D.; Brunner, H.; Oehr, C., Plasma Treatment of Polymers for Surface and Adhesion Improvement. Nuclear Instruments and Methods in Physics Research Section B: Beam Interactions with Materials and Atoms 2003, 208, 281-286.

13. Mata, A.; Fleischman, A. J.; Roy, S., Characterization of Polydimethylsiloxane (PDMS) Properties for Biomedical Micro/Nanosystems. Biomedical Microdevices 2005, 7 (4), 281-293.

14. Kuhr, M.; Bauer, S.; Rothhaar, U.; Wolff, D., Coatings on Plastics with the PICVD Technology. Thin Solid Films 2003, 442 (1-2), 107-116.

15. Favia, P.; Sardella, E.; Gristina, R.; d'Agostino, R., Novel Plasma Processes for Biomaterials: Micro-Scale Patterning of Biomedical Polymers. Surface and Coatings Technology 2003, 169, 707-711.

16. Alissawi, N.; Peter, T.; Strunskus, T.; Ebbert, C.; Grundmeier, G.; Faupel, F., PlasmaPolymerized HMDSO Coatings to Adjust the Silver Ion Release Properties of Ag/Polymer Nanocomposites. Journal of Nanoparticle Research 2013, 15 (11), 2080.

17. Hegemann, D.; Vohrer, U.; Oehr, C.; Riedel, R., Deposition of SiOx Films from $\mathrm{O}_{2} /$ HMDSO Plasmas. Surface and Coatings Technology 1999, 116, 1033-1036.

18. Hegemann, D.; Oehr, C.; Fischer, A., Design of Functional Coatings. Journal of Vacuum Science \& Technology A: Vacuum, Surfaces, and Films 2005, 23 (1), 5-11.

19. Patelli, A.; Vezzu, S.; Zottarel, L.; Menin, E.; Sada, C.; Martucci, A.; Costacurta, S., SiOx-Based Multilayer Barrier Coatings Produced by a Single Pecvd Process. Plasma Processes and Polymers 2009, 6 (S1), S665-S670.

20. Gorshunov, B.; Torgashev, V.; Zhukova, E.; Thomas, V.; Belyanchikov, M.; Kadlec, C.; Kadlec, F.; Savinov, M.; Ostapchuk, T.; Petzelt, J., Incipient Ferroelectricity of Water Molecules Confined to Nano-Channels of Beryl. Nature communications 2016, 7, 12842.

21. Nečas, D.; Klapetek, P., Gwyddion: An Open-Source Software for Spm Data Analysis. Central European Journal of Physics 2012, 10 (1), 181-188.

22. Alexander, M. R.; Short, R.; Jones, F.; Stollenwerk, M.; Zabold, J.; Michaeli, W., An X-Ray Photoelectron Spectroscopic Investigation into the Chemical Structure of Deposits Formed from Hexamethyldisiloxane/Oxygen Plasmas. Journal of Materials Science 1996, 31 (7), 1879-1885.

23. Alexander, M. R.; Short, R. D.; Jones, F. R.; Michaeli, W.; Blomfield, C. J., A Study of $\mathrm{HMDSO} / \mathrm{O}_{2}$ Plasma Deposits Using a High-Sensitivity and -Energy Resolution XPS 
Instrument: Curve Fitting of the Si 2p Core Level. Applied Surface Science 1999, 137 (1), 179183.

24. Paynter, R. W., An Arxps Primer. Journal of Electron Spectroscopy and Related Phenomena 2009, 169 (1), 1-9.

25. Blanchard, N. E.; Naik, V. V.; Geue, T.; Kahle, O.; Hegemann, D.; Heuberger, M., Response of Plasma-Polymerized Hexamethyldisiloxane Films to Aqueous Environments. Langmuir 2015, 31 (47), 12944-12953.

26. Clemens, D.; Gross, P.; Keller, P.; Schlumpf, N.; Könnecke, M., Amor - the Versatile Reflectometer at Sinq. Physica B: Condensed Matter 2000, 276-278, 140-141.

27. Parratt, L. G., Surface Studies of Solids by Total Reflection of X-Rays. Physical review 1954, 95 (2), 359 .

28. Braun, C. The Reflectivity Tool or Parratt32 Version 1.6.0, HMI, Berlin, 1997-2002.

29. Heuberger, M.; Balmer, T. E., The Transmission Interferometric Adsorption Sensor. Journal of Physics D: Applied Physics 2007, 40 (23), 7245.

30. Sannomiya, T.; Balmer, T. E.; Hafner, C.; Heuberger, M.; Vörös, J., Optical Sensing and Determination of Complex Reflection Coefficients of Plasmonic Structures Using Transmission Interferometric Plasmonic Sensor. Review of Scientific Instruments 2010, 81 (5), 053102 .

31. Heuberger, M., The Extended Surface Forces Apparatus. Part I. Fast Spectral Correlation Interferometry. Review of Scientific Instruments 2001, 72 (3), 1700-1707.

32. McMEEKIN, T. L.; Groves, M. L.; Hipp, N. J., Refractive Indices of Amino Acids, Proteins, and Related Substances. ACS Publications: 1964.

33. Barer, R.; Tkaczyk, S., Refractive Index of Concentrated Protein Solutions. Nature 1954, 173 (4409), 821.

34. Borvon, G.; Goullet, A.; Granier, A.; Turban, G., Analysis of Low-K Organosilicon and Low-Density Silica Films Deposited in HMDSO Plasmas. Plasmas and polymers 2002, 7 (4), 341-352.

35. Zajičcková, L.; Burš́́ková, V.; Peřina, V.; Macková, A.; Subedi, D.; Janča, J.; Smirnov, S., Plasma Modification of Polycarbonates. Surface and Coatings Technology 2001, 142, 449454. 
36. Mota, R. P.; Galvão, D.; Durrant, S. F.; De Moraes, M. A. B.; de Oliveira Dantas, S.; Cantão, M., HMDSO Plasma Polymerization and Thin Film Optical Properties. Thin Solid Films 1995, 270 (1-2), 109-113.

37. Hegemann, D., Macroscopic Investigation of Reaction Rates Yielding Plasma Polymer Deposition. Journal of Physics D: Applied Physics 2013, 46 (20), 205204.

38. Korzec, D.; Theirich, D.; Werner, F.; Traub, K.; Engemann, J., Remote and Direct Microwave Plasma Deposition of HMDSO Films: Comparative Study. Surface and Coatings Technology 1995, 74, 67-74.

39. Kaiser, M.; Baumgärtner, K. M.; Mattheus, A., Microwave Plasma Sources-Applications in Industry. Contributions to Plasma Physics 2012, 52 (7), 629-635.

40. Steves, S.; Ozkaya, B.; Liu, C.; Ozcan, O.; Bibinov, N.; Grundmeier, G.; Awakowicz, P., Silicon Oxide Barrier Films Deposited on Pet Foils in Pulsed Plasmas: Influence of Substrate Bias on Deposition Process and Film Properties. Journal of Physics D: Applied Physics 2013, $46(8), 084013$.

41. Haupt, M.; Barz, J.; Oehr, C., Creation and Recombination of Free Radicals in Fluorocarbon Plasma Polymers: An Electron Spin Resonance Study. Plasma Processes and Polymers 2008, 5 (1), 33-43.

42. Granier, A.; Vervloet, M.; Aumaille, K.; Vallée, C., Optical Emission Spectra of Teos and HMDSO Derived Plasmas Used for Thin Film Deposition. Plasma Sources Science and Technology 2003, 12 (1), 89.

43. Kilicaslan, A.; Levasseur, O.; Roy-Garofano, V.; Profili, J.; Moisan, M.; Côté, C.; Sarkissian, A.; Stafford, L., Optical Emission Spectroscopy of Microwave-Plasmas at Atmospheric Pressure Applied to the Growth of Organosilicon and Organotitanium Nanopowders. Journal of Applied Physics 2014, 115 (11), 113301.

44. Deilmann, M.; Halfmann, H.; Steves, S.; Bibinov, N.; Awakowicz, P., Silicon Oxide Permeation Barrier Coating and Plasma Sterilization of Pet Bottles and Foils. Plasma Processes and Polymers 2009, 6 (S1), S695-S699.

45. Wavhal, D. S.; Zhang, J.; Steen, M. L.; Fisher, E. R., Investigation of Gas Phase Species and Deposition of $\mathrm{SiO}_{2}$ Films from $\mathrm{HMDSO} / \mathrm{O}_{2}$ Plasmas. Plasma Processes and Polymers 2006, 3 (3), 276-287.

46. Vautrin-Ul, C.; Roux, F.; Boisse-Laporte, C.; Pastol, J. L.; Chausse, A., Hexamethyldisiloxane (HMDSO)-Plasma-Polymerised Coatings as Primer for Iron Corrosion Protection: Influence of RF Bias. Journal of Materials Chemistry 2002, 12 (8), 2318-2324. 
47. Barni, R.; Zanini, S.; Riccardi, C., Characterization of the Chemical Kinetics in an $\mathrm{O}_{2} /$ HMDSO RF Plasma for Material Processing. Advances in Physical Chemistry 2012, 2012, 6.

48. Kubiak, K.; Wilson, M.; Mathia, T.; Carval, P., Wettability Versus Roughness of Engineering Surfaces. Wear 2011, 271 (3-4), 523-528.

49. Bain, C. D.; Whitesides, G. M., Depth Sensitivity of Wetting: Monolayers of OmegaMercapto Ethers on Gold. Journal of the American Chemical Society 1988, 110 (17), 5897 5898.

50. Azioune, A.; Marcozzi, M.; Revello, V.; Pireaux, J. J., Deposition of Polysiloxane-Like Nanofilms onto an Aluminium Alloy by Plasma Polymerized Hexamethyldisiloxane: Characterization by XPS and Contact Angle Measurements. Surface and Interface Analysis: An International Journal Devoted to the Development and Application of Techniques for the Analysis of Surfaces, Interfaces and Thin Films 2007, 39 (7), 615-623.

51. Nagai, H.; Hori, M.; Goto, T.; Fujii, T.; Hiramatsu, M., Fabrication of Multilayered SiOCH Films with Low Dielectric Constant Employing Layer-by-Layer Process of Plasma Enhanced Chemical Vapor Deposition and Oxidation. Japanese Journal of Applied Physics 2003, 42 (5R), 2775.

52. Sodhi, R. N., Time-of-Flight Secondary Ion Mass Spectrometry (ToF-SIMS):Versatility in Chemical and Imaging Surface Analysis. Analyst 2004, 129 (6), 483-487.

53. Sabata, A.; Van Ooij, W.; Yasuda, H., Plasma-Polymerized Films of Trimethylsilane Deposited on Cold-Rolled Steel Substrates. Part 1. Characterization by XPS, AES and ToF-SIMS. Surface and Interface Analysis 1993, 20 (10), 845-859.

54. Liu, Y.; Zhang, Z.; Wells, M. C.; Beebe, T. P., Growth and Reactions of SiOx/Si Nanostructures on Surface-Templated Molecule Corrals. Langmuir 2005, 21 (19), 8883-8891. 55. Lazauskas, A.; Baltrusaitis, J.; Grigaliūnas, V.; Jucius, D.; Guobienè, A.; Prosyčevas, I.; Narmontas, P., Characterization of Plasma Polymerized Hexamethyldisiloxane Films Prepared by Arc Discharge. Plasma Chemistry and Plasma Processing 2014, 34 (2), 271-285. 56. Creatore, M.; Palumbo, F.; d'Agostino, R., Deposition of SiOx Films from Hexamethyldisiloxane/Oxygen Radiofrequency Glow Discharges: Process Optimization by Plasma Diagnostics. Plasmas and Polymers 2002, 7 (3), 291-310.

57. Rau, C.; Kulisch, W., Mechanisms of Plasma Polymerization of Various Silico-Organic Monomers. Thin Solid Films 1994, 249 (1), 28-37. 
58. Vickerman, J. C.; Briggs, D., ToF-SIMS: Materials Analysis by Mass Spectrometry. Surface Spectra: 2013.

59. Zuber, K.; Markanday, J. F. S.; Hall, C.; Evans, D.; Charrault, E.; Murphy, P., PostPolymerization Surface Segregation in Thin Pecvd Siloxane Films Leading to a SelfRegenerative Effect. Plasma Processes and Polymers 2017, 14 (9), 1600233.

60. Mahoney, C. M., Cluster Secondary Ion Mass Spectrometry of Polymers and Related Materials. Mass Spectrometry Reviews 2010, 29 (2), 247-293.

61. D. J. O`Connor, B. A. S., Surface Analysis Methods in Materials Science. 2 ed.; Springer-Verlag Berlin: 2003.

62. Guermat, N.; Bellel, A.; Sahli, S.; Segui, Y.; Raynaud, P., Thin Plasma-Polymerized Layers of Hexamethyldisiloxane for Humidity Sensor Development. Thin Solid Films 2009, $517(15), 4455-4460$.

63. Doremus, R. H., Diffusion of Water in Silica Glass. Journal of Materials Research 1995, $10(9), 2379-2389$.

64. Erlat, A.; Spontak, R.; Clarke, R.; Robinson, T.; Haaland, P.; Tropsha, Y.; Harvey, N.; Vogler, E., SiOx Gas Barrier Coatings on Polymer Substrates: Morphology and Gas Transport Considerations. The Journal of Physical Chemistry B 1999, 103 (29), 6047-6055.

65. Singh, K.; Scholl, H.; Brinkmann, M.; Di Michiel, M.; Scheel, M.; Herminghaus, S.; Seemann, R., The Role of Local Instabilities in Fluid Invasion into Permeable Media. Scientific Reports 2017, 7 (1), 444.

66. Chang, T.; Mor, Y.; Liu, P.; Tsai, T.; Chen, C.; Mei, Y.; Sze, S., Recovering Dielectric Loss of Low Dielectric Constant Organic Siloxane During the Photoresist Removal Process. Journal of the Electrochemical Society 2002, 149 (8), F81-F84.

67. Horner, A.; Zocher, F.; Preiner, J.; Ollinger, N.; Siligan, C.; Akimov, S. A.; Pohl, P., The Mobility of Single-File Water Molecules is Governed by the Number of H-Bonds They May Form with Channel-Lining Residues. Science Advances 2015, 1 (2).

68. Moser, P.; Squire, P.; O'Konski, C., Electric Polarization in Proteins-Dielectric Dispersion and Kerr Effect Studies of Isoionic Bovine Serum Albumin. The Journal of Physical Chemistry 1966, 70 (3), 744-756.

69. Hähl, H.; Evers, F.; Grandthyll, S.; Paulus, M.; Sternemann, C.; Loskill, P.; Lessel, M.; Hüsecken, A. K.; Brenner, T.; Tolan, M.; Jacobs, K., Subsurface Influence on the Structure of Protein Adsorbates as Revealed by in Situ X-Ray Reflectivity. Langmuir 2012, 28 (20), 7747-7756. 


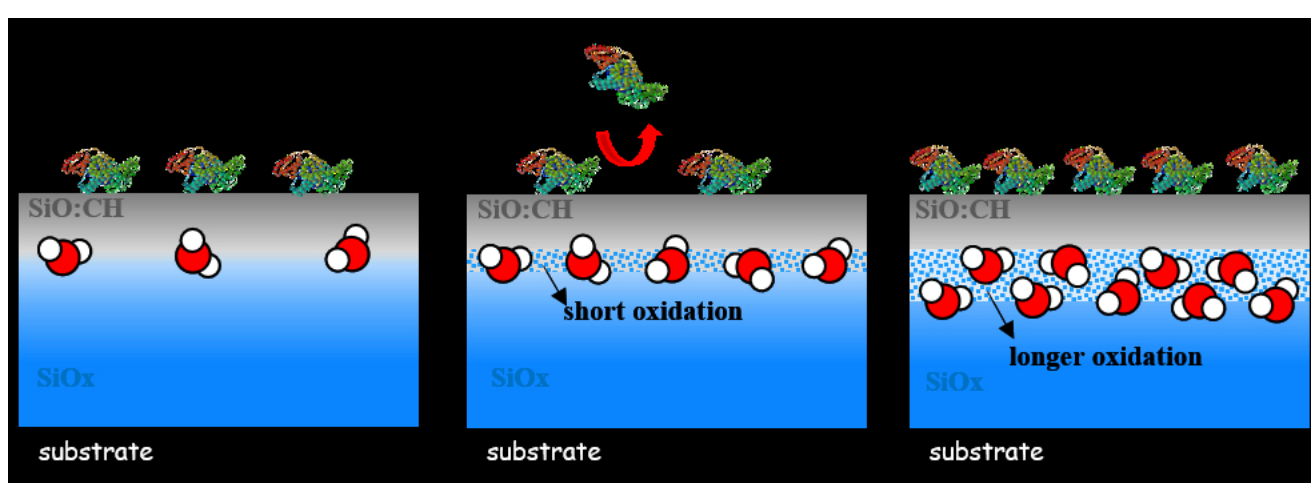

$165 \times 58 \mathrm{~mm}(150 \times 150 \mathrm{DPI})$ 